\title{
Renormalization Group
}

\section{Flows from Holography;}

\author{
Supersymmetry \\ and a c-Theorem
}

\author{
D. Z. Freedman ${ }^{a}$, S. S. Gubser ${ }^{b}$, K. Pilch ${ }^{c}$ and N. P. Warner ${ }^{d}$ \\ ${ }^{a}$ Department of Mathematics and Center for Theoretical Physics, \\ Massachusetts Institute of Technology, \\ Cambridge, MA 02139 \\ ${ }^{b}$ Department of Physics, \\ Harvard University, \\ Cambridge, MA 02138, USA \\ ${ }^{c}$ Department of Physics and Astronomy, \\ University of Southern California, Los Angeles, \\ CA 90089-0484, USA \\ ${ }^{d}$ Theory Division, CERN, \\ CH-1211 Geneva 23, Switzerland
}

\begin{abstract}
We obtain first order equations that determine a supersymmetric kink solution in five-dimensional $\mathcal{N}=8$ gauged supergravity. The kink interpolates between an exterior anti-de Sitter region with maximal supersymmetry and an interior anti-de Sitter region with one quarter of the maximal supersymmetry. One eighth of supersymmetry is preserved by the kink as a whole. We interpret it as describing the renormalization group flow in $\mathcal{N}=4$ super-Yang-Mills theory broken to an $\mathcal{N}=1$ theory by the addition of a mass term for one of the three adjoint chiral superfields. A detailed correspondence is obtained between fields of bulk supergravity in the interior anti-de Sitter region and composite operators of the infrared field theory. We also point out that the truncation used to find the reduced symmetry critical point can be extended to obtain a new $\mathcal{N}=4$ gauged supergravity theory holographically dual to a sector of $\mathcal{N}=2$ gauge theories based on quiver diagrams.
\end{abstract}


We consider more general kink geometries and construct a c-function that is positive and monotonic if a weak energy condition holds in the bulk gravity theory. For even-dimensional boundaries, the c-function coincides with the trace anomaly coefficients of the holographically related field theory in limits where conformal invariance is recovered.

\section{Introduction}

The conjecture [1] of the equivalence of string theory on $A d S_{5} \times S^{5}$ to $\mathcal{N}=4$ supersymmetric Yang-Mills theory in $(3+1)$-dimensions derived some of its initial motivation and plausibility from the $S U(2,2 \mid 4)$ superconformal invariance of both sides of the equivalence. Optimistically, one might expect that the strategy for computing Green's functions $[2,3]$ is more general, and applies to any situation where a quantum field theory (QFT) on the boundary of a spacetime can be related to properties of a quantum gravity theory in the bulk. To test this expectation it would be good to have an example of a QFT-gravity pair where the QFT is non-conformal but nevertheless well-understood at least in some aspects which can be probed from the gravity side. Furthermore, the bulk description should be in terms of a geometry in which the supergravity approximation to string theory is uniformly applicable.

The main purpose of this paper is to provide such an example. The QFT will be the $\mathcal{N}=1$ supersymmetric gauge theory obtained from $\mathcal{N}=4$ superYang-Mills by adding a mass to one of the three chiral adjoint superfields. The mass breaks conformal invariance and drives a renormalization group flow. Using the methods developed by Leigh and Strassler [4] one can argue that the theory recovers superconformal invariance in the infrared, and that in fact there is a line of fixed points in the infrared as well as the ultraviolet. The theory can be made strongly coupled in both limits by making the ultraviolet 't Hooft coupling large, and so a supergravity description at large $N$ should be possible.

We believe that the supergravity dual of this field theory flow is the kink solution that is discussed in section 5 , and which interpolates between the AdS geometries at two critical points of five-dimensional $\mathcal{N}=8$ gauged supergravity $[5,6,7]$. The critical point describing the UV end of the flow is the expected maximally supersymmetric vacuum of the bulk theory, which is dual to $\mathcal{N}=4 \mathrm{SYM}$. The kink itself preserves four real supercharges, as does the field theory described in the previous paragraph. In the IR limit the kink approaches the critical point with $\mathcal{N}=2$ bulk supersymmetry that was discovered in [8]. 
The most direct evidence for the correspondence between the field theory flow and the supergravity kink is that the symmetries match. Along the whole RG flow there is $S U(2) \times U(1)$ global symmetry, and this symmetry is also present in the kink. The AdS geometries at the endpoints imply conformal symmetry in the UV and IR limits of the field theory, and there is $S U(2,2 \mid 4)$ symmetry at the UV end and $S U(2,2 \mid 1)$ symmetry at the IR end. Furthermore, the limiting behavior of the kink near its UV endpoint involves scalars with the right quantum numbers and dimensions to be dual to the mass term for one adjoint chiral superfield. There is additional evidence from the match of trace anomaly coefficients computed at both endpoints in field theory and through the AdS/CFT correspondence. The authors of [9] independently considered the field theory RG flow and the matching of trace anomaly coefficients.

To probe this correspondence more deeply, we compute mass eigenvalues of all fields of gauged supergravity at the non-trivial supersymmetric critical point. We use this and the results of $[2,3]$ to obtain the scaling dimensions of the corresponding gauge theory operators. The details are presented in section 6 and in an appendix. Boson and fermion operators neatly combine into multiplets that are representations of the superalgebra $S U(2,2 \mid 1)$ of $\mathcal{N}=1$ superconformal symmetry in four dimensions. We exhibit gauge invariant combinations of the massless superfields of the gauge theory whose scaling dimensions and $S U(2) \times U(1)$ quantum numbers precisely match the five short multiplets observed in supergravity. There are three additional long multiplets which complete the picture. This detailed field-operator map constitutes perhaps the strongest evidence that the supergravity critical point is indeed the holographic dual of the mass-deformed $\mathcal{N}=4$ theory.

Non-conformal examples of the bulk-boundary holographic relation already exist in the literature (see for instance $[10,11,12,13,14,15,16]$ ), but from the point of view of testing the duality most of these are vexed either by an incomplete understanding of the boundary theory, or by a bulk geometry where supergravity is unreliable due to tachyons, or large curvatures, or a strong dilaton - or some combination of these.

Our example is closer to the work of $[17,18,19,20,21]$, and we draw on various aspects of these papers in our analysis. In [17] it was proposed that a blowup of an $S^{5} / \mathbf{Z}_{2}$ orbifold, deformed to a coset manifold $S O(4) / S O(2)$, described the flow of an $\mathcal{N}=2$ super-Yang-Mills theory to an $\mathcal{N}=1$ infrared fixed point through the addition of a mass term. The dual field to this mass term was a twisted string state, so a direct analysis of the flow geometry beyond the level of topology seemed difficult. Similar examples were considered in $[18,19]$, and in [18] it was suggested that the flows could 
be described in terms of tensor multiplets of an $\mathcal{N}=4$ gauged supergravity. This was motivated in part by the work of $[20,21]$, where anti-de Sitter vacua of $\mathcal{N}=8, d=5$ gauged supergravity discovered in [6] were re-examined as candidate descriptions of non-supersymmetric fixed points of RG flows. The main example considered in $[20,21]$ was a $S U(3)$-symmetric point. Second order differential equations were found for a five-dimensional flow geometry inteperpolating between the maximally supersymmetric point and the $S U(3)$-symmetric one. The field theory interpretation was that adding a mass to one of the four gauginos resulted in a flow to an infrared fixed point of RG.

A general problem of studying RG flows through the bulk-boundary correspondence is that the validity of supergravity depends on having strong coupling in the gauge theory, so that results cannot be compared with perturbative treatments of the renormalization group flow. For instance, it is difficult to verify from a field theory point of view any of the properties of the $S U(3)$-symmetric critical point studied in [20,21]. What allows us to make non-trivial field theory predictions about the infrared fixed point is the supersymmetry of the fixed point and the $\mathcal{N}=1$ supersymmetry that must be preserved throughout the flow. These predictions are analogous to those which have been checked in the conifold examples $[17,22,18,19]$. What is new in our paper as compared to these is that we are able to give an explicit five-dimensional supergravity description of a supersymmetric flow geometry.

Before restricting our attention to a particular example, we consider in section 4 some general properties of the supergravity kinks that could be used to describe renormalization group flows. We are able to identify a monotonic function along the kink which interpolates between the anomaly coefficients at one end and at the other. Using nothing more than Einstein's equations and a weak energy condition it is possible to show that such a function can always be found in any kink geometry with the Poincaré symmetries of the boundary theory in flat space. The argument is dimension-independent. Thus, on rather general grounds, the holographic bulk-boundary correspondence implies a c-theorem, at least in the regime where classical gravity is a reliable guide to the bulk physics. In the four-dimensional case, an equivalent c-function was discussed independently in [20], and its monotonicity was checked using the equations of motion for the supergravity geometries considered there.

In section 5 we find the supergravity kink corresponding to the supersymmetric renormalization group flow described above. We do this by demanding that one eighth of supersymmetry is unbroken throughout the bulk, 
and we thus obtain first order equations for a kink solution that interpolates between the maximally supersymmetric critical point and the one-quarter supersymmetric point of [8]. The first order equations for the scalar fields are the gradient flow equations of a "superpotential" defined on a restricted four-dimensional slice of the scalar manifold, and simply related to the potential of gauged supergavity on this slice.

We work within the framework of gauged $\mathcal{N}=8$ supergravity in five dimensions because its full non-linear structure is known. This is not the case for the complete Kaluza-Klein reduction of ten-dimensional type IIB supergravity on $S^{5}$. The spectrum of five-dimensional $\mathcal{N}=8$ gauged supergravity includes only the first few Kaluza-Klein modes of the ten-dimensional theory, but it is a complete theory at the classical level and was argued in [8] to be a consistent truncation of the parent theory. This has not been proven explicitly but is expected after suitable field redefinitions of the heavy KK modes. Consistent truncation means that any solution of the truncated theory can be lifted to a solution of the untruncated theory.

Another reason for working with five-dimensional gauged supergravity is that it efficiently encodes a class of metric deformations of the $S^{5}$, and a family of backgrounds for $B_{M N}^{N S, R R}$ fields where the indices lie in the $S^{5}$ directions. To be more specific, the five-dimensional gauged supergravity has 42 scalar fields that parameterize the coset $E_{6(6)} / U S p(8)$. From the holographic perspective two of these scalars correspond to the Yang-Mills gauge coupling and $\theta$-angle, 20 of them parametrize Yang-Mills scalar masses (deformations of the metric on $S^{5}$ ), and the other 20 parametrize the possible Yang-Mills fermion masses (special backgrounds for $B_{M N}^{N S, R R}$ ). Indeed gauged $\mathcal{N}=8$ supergravity in five dimensions is holographically dual to the YangMills energy-momentum tensor supermultiplet, and the supergravity scalars are the moduli of this supermultiplet: the couplings and the masses.

The lagrangian of gauged $\mathcal{N}=8$ supergravity has a scalar potential $V$ which is invariant under the gauged subalgebra, $S O(6)=S U(4)$, of $E_{6(6)}$. Critical points of the potential $V$ give rise to $A d S_{5}$ solutions of gauged supergravity. The list of known critical points can be found in [8]. For some of them the corresponding ten-dimensional geometry can be found in the literature: for the $S U(3)$ symmetric points see [23]; for the $S O(5)$ symmetric points see [24]. The critical point which we claim describes the infrared endpoint of our flow is the recently discovered $S U(2) \times U(1)$-symmetric point with one-quarter supersymmetry [8]. The corresponding ten-dimensional geometry is, as yet, not known, but its metric can be computed using the results of [8]. 
The known critical points of [8] were found by specializing to an elevendimensional, truncated submanifold, $\mathcal{S}$, of $E_{6(6)} / U S p(8)$. This is the invariant subspace under a particular $S U(2)$ subgroup of $S O(6)=S U(4)$. Since this scalar submanifold will play a major role in our work we will review its structure in detail in section 3 and in two appendices.

In section 7 we point out that the truncation of the scalar manifold can be extended to the full theory and leads to a complete five-dimensional $\mathcal{N}=4$ gauged supergravity theory coupled to two tensor multiplets. We suggest that this theory captures a sector common to all $\mathcal{N}=2$ superconformal gauge theories based on quiver diagrams $[25,26]$, and that the kink we have found may reflect certain renormalization group flows in these theories as well.

A comment on the counting of supersymmetries may prevent future confusion. A five-dimensional supergravity theory is referred to as having $\mathcal{N}$ extended supersymmetry if the invariance of the lagrangian is characterized by $4 \mathcal{N}$ real supercharges, and similarly with a background of supergravity. The minimally supersymmetric five-dimensional supergravity has $\mathcal{N}=2$ in the lagrangian. As always, maximal supersymmetry is 32 real supercharges. A four-dimension field theory with $\mathcal{N}$-extended supersymmetry also has $4 \mathcal{N}$ real supercharges, unless it also has conformal symmetry, which doubles the number of supercharges to $8 \mathcal{N}$. In an effort to speak French to the French and English to the Americans, we will refer to a supergravity background dual to an $\mathcal{N}=1$ superconformal field theory as $\mathcal{N}=2$.

\section{Field theory motivation}

Asymptotic freedom and confinement are difficult to capture reliably in a supergravity geometry. For asymptotic freedom this is because the 't Hooft coupling eventually gets weak in the ultraviolet, and $\alpha^{\prime}$ corrections become important to any supergravity description. All geometric attempts to describe confinement include singularities (some more malignant than others), and there is a variety of problems regarding the discrepancy between the confining string tension and the mass gap, nearly flat Regge trajectories, and unwanted global symmetries. The study of renormalization group flows in supersymmetric gauge theories provides us with some examples where both the ultraviolet and the infrared fixed points are conformal, and neither asymptotic freedom nor confinement is encountered.

The example upon which we want to focus is a relevant deformation of 
$\mathcal{N}=4$ super-Yang-Mills theory by a mass term for one of the three $\mathcal{N}=1$ adjoint chiral superfields which, together with the $\mathcal{N}=1$ adjoint vector superfield, fill out the full $\mathcal{N}=4$ gauge multiplet. In $\mathcal{N}=1$ language, the superpotential is

$$
W=\operatorname{Tr} \Phi_{3}\left[\Phi_{1}, \Phi_{2}\right]+\frac{1}{2} m \operatorname{Tr} \Phi_{3}^{2} .
$$

The first term is the superpotential required by $\mathcal{N}=4$ supersymmetry. The second term breaks $\mathcal{N}=4$ down to $\mathcal{N}=1$, and also destroys conformal invariance, so the total number of real supercharges is reduced from thirtytwo to four. The theory does have a non-trivial infrared fixed point. Indeed, it was shown in [4] that by choosing the anomalous dimensions $\gamma_{1}=\gamma_{2}=$ $-1 / 4$ and $\gamma_{3}=1 / 2$ for $\Phi_{1}, \Phi_{2}$, and $\Phi_{3}$, one could simultaneously make the NSVZ exact beta function [27],

$$
\beta(g)=-\frac{g^{3} N_{c}}{8 \pi^{2}} \frac{3-\sum_{i}\left(1-2 \gamma_{i}\right)}{1-\frac{g^{2} N_{c}}{8 \pi^{2}}},
$$

vanish, and make the superpotential in 2.1 dimension three, which is marginal. Note that it is immaterial in this analysis whether one integrates out $\Phi_{3}$ or not. The theory we have described has some features in common with $\mathcal{N}=1$ SQCD with $N_{f}=2 N_{c}$, and it is its own Seiberg dual.

That the infrared fixed point of this flow is the holographic dual theory to the one-quarter supersymmetric vacuum found in [8] seems to have been observed independently by several groups, including the present authors, the authors of [9], and M. Strassler [28]. For us the crucial observation was that the coefficients in the trace anomaly predicted by field theory match those observed in supergravity. The coefficients we aim to calculate are $a$ and $c$ in the one anomalous point functions which in the notation of [29] read

$$
\begin{aligned}
\left\langle T_{\mu}^{\mu}\right\rangle_{g_{\mu \nu}, V_{\mu}} & =\frac{c}{16 \pi^{2}} W_{\mu \nu \rho \sigma}^{2}-\frac{a}{16 \pi^{2}} \tilde{R}_{\mu \nu \rho \sigma} \tilde{R}^{\mu \nu \rho \sigma}+\frac{c}{6 \pi^{2}} V_{\mu \nu}^{2} \\
\left\langle\partial_{\mu} \sqrt{g} R^{\mu}\right\rangle_{g_{\mu \nu}, V_{\mu}} & =-\frac{a-c}{24 \pi^{2}} R_{\mu \nu \rho \sigma} \tilde{R}^{\mu \nu \rho \sigma}+\frac{5 a-3 c}{9 \pi^{2}} V_{\mu \nu} \tilde{V}^{\mu \nu}
\end{aligned}
$$

in the presence of a metric $g_{\mu \nu}$ and a source $V_{\mu}$ for the R-current $R^{\mu}$. The normalization of $a$ and $c$ is such that $c=1 / 120$ for a single free, real scalar, and $c=\left(N_{c}^{2}-1\right) / 4$ for $S U\left(N_{c}\right)$ super-Yang-Mills. The combinations $a-c$ and $5 a-3 c$ in the second line are consequences of $\mathcal{N}=1$ supersymmetry [29]. In the ultraviolet, where we can effectively set $m_{3}=0$, we arrive at $a-c=0$ and $5 a-3 c \propto \frac{8}{9}\left(N_{c}^{2}-1\right)$ by inspection of the triangle diagrams in Figure 1. The current $R_{\mu}$ assigns $r(\lambda)=1$ to the gauginos and $r(\psi)=-1 / 3$ to the quarks.

When $m \neq 0$, this R-current becomes anomalous, which is entirely appropriate since $\partial_{\mu} R^{\mu}$ is an $\mathcal{N}=1$ superpartner of $T_{\mu}^{\mu}$, and the theory now 
is changing with scale. There is however a non-anomalous combination [30] of $R_{\mu}$ with the Konishi currents, $K_{\mu}^{i}$. By definition, $K_{\mu}^{i}$ assigns charge 1 to the fields in the $i^{\text {th }}$ chiral multiplet and charge 0 to the fields in vector multiplets. The non-anomalous current is

$$
S_{\mu}=R_{\mu}+\frac{2}{3} \sum_{i}\left(\gamma_{\mathrm{IR}}^{i}-\gamma^{i}\right) K_{\mu}^{i}
$$

Here $\gamma^{i}$ is the anomalous dimension of the $i^{\text {th }}$ chiral superfield, and the values of $\gamma_{\mathrm{IR}}^{i}$ are those discussed in the text preceding 2.2. 'T Hooft anomaly matching implies that $\left\langle\partial_{\mu} R^{\mu}\right\rangle$ in the infrared is equal to $\left\langle\partial_{\mu} S^{\mu}\right\rangle$ in the ultraviolet. In the ultraviolet, $\gamma^{i}=0$ and the perturbative analysis in terms of fermions running around a loop can be applied. The result is $a-c=0$ and $5 a-3 c=\frac{3}{4}\left(N_{c}^{2}-1\right)$ with the same constant of proportionality as before. Combining with the results of the previous paragraph, one finds

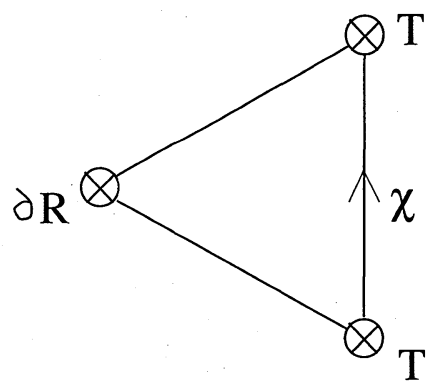

$$
a-c \propto \sum_{\chi} r(\chi)
$$

a)

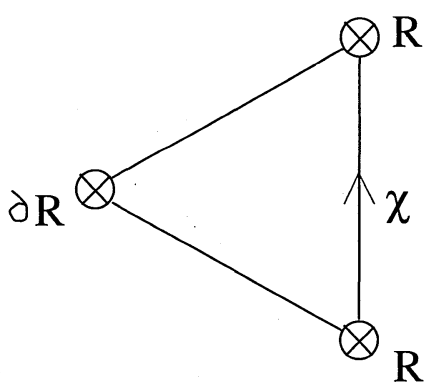

$5 a-3 c \propto \sum_{\chi} r(\chi)^{3}$

b)

Fig. 1: Triangle diagrams for computing the anomalous contribution to $\partial_{\mu} R^{\mu}$. The sum is over the chiral fermions $\chi$ which run around the loop, and $r(\chi)$ is the R-charge of each such fermion.

$$
\frac{a_{\mathrm{IR}}}{a_{\mathrm{UV}}}=\frac{c_{\mathrm{IR}}}{c_{\mathrm{UV}}}=\frac{27}{32} .
$$

The non-anomalous $S_{\mu}$ current generates an exact symmetry of the massdeformed theory, and it will be matched precisely by a $U(1)$ symmetry of the supergravity kink solution. This symmetry is called $U(1)_{R}$ in subsequent sections.

From the gravity side it is more straightforward to compute $\left\langle T_{\mu}^{\mu}\right\rangle$, since 
we can rely on the analysis of [31]. We remain in mostly minus conventions. Suppose we are given any compactification of string theory or M-theory (or any other, as-yet-unknown theory of quantum gravity) whose non-compact portion is $A d S_{5}$ with $R_{\mu \nu}=\Lambda g_{\mu \nu}$. If we rescale the metric by a factor of $4 / \Lambda$, we obtain the dimensionless $A d S_{5}$ metric $\widehat{d s}_{5}^{2}$ with $\hat{R}_{\mu \nu}=4 \hat{g}_{\mu \nu}$. In defining a conformal field theory through its duality to the $A d S_{5}$ compactification under consideration, the part of the action relevant to the computation of $\left\langle T_{\mu}^{\mu}\right\rangle$ is the Einstein-Hilbert term plus the cosmological term:

$$
S=-\frac{1}{2 \kappa_{5}^{2}} \int d^{5} x \sqrt{g}(R-3 \Lambda+\ldots)=-\frac{4}{\kappa_{5}^{2} \Lambda^{3 / 2}} \int d^{5} x \sqrt{\hat{g}}(\hat{R}-12+\ldots)
$$

where $\kappa_{5}^{2}=8 \pi G_{5}$ is the five-dimensional gravitational coupling. Directly from [31] we read off

$$
a=c=\frac{\pi}{G_{5} \Lambda^{3 / 2}} .
$$

In solutions of $\mathcal{N}=8$ supergravity where the scalars are fixed, $\Lambda=-\frac{4}{3} V$. It was commented on in [8] already that

$$
\frac{c_{\mathrm{IR}}}{c_{\mathrm{UV}}}=\left(\frac{V_{\mathrm{UV}}}{V_{\mathrm{IR}}}\right)^{-3 / 2}=\frac{27}{32} \text {. }
$$

The agreement betwen 2.5 and 2.8 is a sign that we have found the correct field theory interpretation of the infrared AdS region. It is straightforward to check the overall normalization of $a$ and $c: 2.7$ leads to $a_{U V}=c_{U V}=N^{2} / 4$, which differs from the $S U(N)$ gauge theory result only by a $1 / N^{2}$ correction. This agreement can be traced back to absorption calculations [32].

The essentials of the anomaly-matching analysis were worked out in [22] for the conifold theory introduced in [17]. Aspects of it were also employed in [21] and in particular in [9] in a field theory analysis independent of our own for the one-quarter supersymmetric fixed point of [8].

The fields involved in the interpolating solution between the maximally supersymmetric anti-de Sitter vacuum of gauged supergravity and the onequarter supersymmetric vacuum have masses near the maximally supersymmetric end which correspond to operators of dimensions 2 and 3 . These are, respectively, the boson and fermion mass involved in the second term of 2.1. Appropriately, turning on the scalar dual to the boson mass term preserves $S O(4) \times S O(2) \subset S O(6)$, while turning on the scalar dual to the fermion mass term preserves $S U(3)$. Together they break the symmetry down to $S U(2) \times U(1)$. If one regards the presence of these scalars in the interpolating solution, plus the supersymmetry, plus the existence of the interior AdS region, as sufficient evidence that the infrared fixed point in field theory 
is described by the deformation of $\mathcal{N}=4$ super-Yang-Mills which we have discussed above, then the agreement between 2.5 and 2.8 can be regarded as a successful field theory prediction of the value of the scalar potential $V$ at the saddle point that defines this solution. From this point of view, testing dimensions of scalar operators in field theory against masses of gauged supergravity fields amounts to checking field theory predictions of the second derivatives of $V$ at the saddle point.

\section{$3 \mathcal{N}=8$ Supergravity and conformal phases of Yang-Mills theory}

All the 42 scalars in five-dimensional $\mathcal{N}=8$ gauged supergravity participate in the scalar potential except for the dilaton and the axion. In view of the correspondence to operators in the Yang-Mills theory explained in the introduction, critical points of this potential correspond to conformal fixed points of $\mathcal{N}=4$ Yang-Mills theory obtained by turning (Yang-Mills) fermion and scalar masses and running to the infrared.

In an effort to classify some of the new phases of the Yang-Mills theory the authors of [8] classified all critical points of the $\mathcal{N}=8$ gauged supergravity potential preserving at least a particular $S U(2)$ symmetry. The initial motivation for this was largely pragmatic: reducing the problem of forty two scalars to a solvable one involving only eleven. As we will explain in more detail in section 7, the truncation to $S U(2)$ singlets can be extended to the whole $\mathcal{N}=8$ theory, and the result is a gauged $\mathcal{N}=4$ supergravity theory coupled to two tensor multiplets. We believe that this truncated theory is holographically dual to a subsector of operators which is common to all $\mathcal{N}=2$ super-Yang-Mills theories based upon quiver diagrams.

The purpose of this section is to review the analysis in [8] of critical points in supergravity with particular attention to the one that preserves one quarter of supersymmetry. This critical point is a reasonable candidate for the description of the IR fixed point of the field theory flow described in section 2 . We begin in section 3.1 by summarizing the $S U(2)$ singlet sector of the supergravity scalars. We also define a sort of superpotential which is helpful for the analysis of supersymmetry. We summarize this analysis at the critical point in section 3.2, and we extend it to non-anti-de Sitter geometries in section 5 . 


\subsection{The scalar structure of the $S U(2)$ singlet sector}

The $\mathcal{N}=8$ supergravity theory has a gauge symmetry of $S U(4)$ or $S O(6)$. We consider the $S U(2)_{I} \times S U(2)_{G} \times U(1)_{G}$ subgroup of $S U(4)$, and look at the singlets of $S U(2)_{I}$. (The subscripts $I$ and $G$ on the groups are intended to distinguish the "Invariance" group from the residual "Gauge" group.) As was discussed in [8], these scalar singlets may be thought of as parametrizing the coset:

$$
\mathcal{S}=\frac{S O(5,2)}{S O(5) \times S O(2)} \times O(1,1) .
$$

The group $S O(5,2)$ contains an obvious $S O(3) \times S O(2,2)$ subgroup, and $S O(2,2)=S L(2, \mathbb{R}) \times S L(2, \mathbb{R})$. The $S O(3)$ is $S U(2)_{G}$, one $S L(2, \mathbb{R})$ describes the dilaton and axion of the theory, and $U(1)_{G}$ is the compact subgroup of the other $S L(2, \mathbb{R})$.

The scalar potential, $V$, on the eleven-dimenensional scalar manifold, $\mathcal{S}$, has a residual $S U(2) \times U(1) \times S L(2, \mathbb{R})$ invariance and can therefore be parametrized in terms of four real variables [8]:

$$
\begin{aligned}
V= & -\frac{g^{2}}{4}\left[\rho^{-4}\left(1-\cos ^{2}(2 \phi)\left(\sinh ^{2}\left(\varphi_{1}\right)-\sinh ^{2}\left(\varphi_{2}\right)\right)^{2}\right)\right. \\
& +\rho^{2}\left(\cosh \left(2 \varphi_{1}\right)+\cosh \left(2 \varphi_{2}\right)\right)+\frac{1}{16} \rho^{8}\left(2+2 \sin ^{2}(2 \phi)\right. \\
& \left.\left.-2 \sin ^{2}(2 \phi) \cosh \left(2\left(\varphi_{1}-\varphi_{2}\right)\right)-\cosh \left(4 \varphi_{1}\right)-\cosh \left(4 \varphi_{2}\right)\right)\right]
\end{aligned}
$$

Our variables are related to those of [8] by $r_{x}=-\left(\varphi_{1}-\varphi_{2}\right) / 2, r_{y}=\left(\varphi_{1}+\right.$ $\left.\varphi_{2}\right) / 2$, and $\theta=2 \phi$. As in [8], $\rho=e^{\alpha}$, parametrizes one sheet of the $O(1,1)$ factor of 3.1.

The $\mathcal{N}=8$ supergravity has a $U S p(8)$ invariance, and in particular the scalar structure is encoded in two $U S p(8)$ tensors, $W_{a b}$ and $A_{a b c d}$. The truncation of the $U S p(8)$ indices to the singlets of $S U(2)_{I}$ can be effected by projecting the 8 of $U S p(8)$ onto the space spanned by the four vectors

$$
\begin{array}{ll}
v_{1}=(1,0,0,0,0,1,0,0), & \tilde{v}_{1}=(0,1,0,0,-1,0,0,0), \\
v_{2}=(0,0,0,1,0,0,1,0), & \tilde{v}_{2}=(0,0,1,0,0,0,0,-1) .
\end{array}
$$

These vectors also turn out to be global eigenvectors of the tensor $W_{a b}$, with eigenvalues, $\lambda_{1}, \bar{\lambda}_{1}$ and $\lambda_{2}, \bar{\lambda}_{2}$ respectively, where

$$
\begin{aligned}
\lambda_{1}= & -\frac{e^{-2 i \phi}}{4 \rho^{2}}\left[\rho ^ { 6 } \left(2 \cos (2 \phi)-\cosh \left(2 \varphi_{1}\right)+\cosh \left(2 \varphi_{2}\right)\right.\right. \\
& \left.+2 i \sin (2 \phi) \cosh \left(\varphi_{1}-\varphi_{2}\right)\right) \\
& +\left(2 \cos (2 \phi)\left(\cosh \left(2 \varphi_{1}\right)+\cosh \left(2 \varphi_{2}\right)\right)\right.
\end{aligned}
$$




$$
\begin{aligned}
& \left.\left.+4 i \sin (2 \phi) \cosh \left(\varphi_{1}+\varphi_{2}\right)\right)\right] \\
\lambda_{2}=- & \frac{e^{-2 i \phi}}{4 \rho^{2}}\left[\rho ^ { 6 } \left(2 \cos (2 \phi)+\cosh \left(2 \varphi_{1}\right)-\cosh \left(2 \varphi_{2}\right)\right.\right. \\
& \left.+2 i \sin (2 \phi) \cosh \left(\varphi_{1}-\varphi_{2}\right)\right) \\
& +\left(2 \cos (2 \phi)\left(\cosh \left(2 \varphi_{1}\right)+\cosh \left(2 \varphi_{2}\right)\right)\right. \\
& \left.\left.+4 i \sin (2 \phi) \cosh \left(\varphi_{1}+\varphi_{2}\right)\right)\right] .
\end{aligned}
$$

These eigenvalues also provide a superpotential $W$ related to $V$ by

$$
V=\frac{g^{2}}{8} \sum_{j=1}^{3}\left|\frac{\partial W}{\partial \varphi_{j}}\right|^{2}-\frac{g^{2}}{3}|W|^{2}
$$

where $\varphi_{3}=\sqrt{6} \alpha$, and $W=\lambda_{1}$ or $W=\lambda_{2}$. We use the metric $\delta_{i j}$ to contract the indices of the partial derivatives of $W$ with respect to $\varphi_{i}$, and as we will see, this is the proper metric on this part of the scalar manifold.

The scalar and gravity part of the $\mathcal{N}=8$ supergravity action is [6]:

$$
\int d^{5} x \sqrt{|g|}\left[-\frac{1}{4} R+\frac{1}{24} g^{\alpha \beta} P_{\alpha a b c d} P_{\beta}^{a b c d}-V\right] .
$$

In 3.6 and in the rest of this paper, we are working in five-dimensional Planck units such that $\kappa_{5}^{2}=2$. The efficient way to repristinate Newton's constant is to insert $2 / \kappa_{5}^{2}$ as an overall factor in from of 3.6: that way the equations of motion are not changed. In particular, the maximally supersymmetric $A d S_{5}$ vacuum of the theory is determined by the equation

$$
R_{\alpha \beta}=-\frac{4}{3} V_{0} g_{\alpha \beta}=g^{2} g_{\alpha \beta}
$$

Comparison with the usual form, $R_{\alpha \beta}=\frac{4}{L^{2}} g_{\alpha \beta}$, yields $g=2 / L$. In the $A d S_{5} \times S^{5}$ vacuum of type IIB theory supported by $N$ units of self-dual five-form flux, $L$ enters as the radius of the $S^{5}$, and one has the standard relation

$$
L^{4}=\frac{\kappa_{10} N}{2 \pi^{5 / 2}} .
$$

In the foregoing parametrization of the scalar manifold, the scalar kinetic term of [6] reduces to:

$$
\begin{aligned}
\frac{1}{24} g^{\mu \nu}\left(P_{\mu a b c d} P_{\nu}^{a b c d}\right)= & \frac{1}{2}\left[\sum_{j=1}^{3} g^{\mu \nu}\left(\partial_{\mu} \varphi_{j}\right)\left(\partial_{\nu} \varphi_{j}\right)\right] \\
& +\sinh ^{2}\left(\varphi_{1}-\varphi_{2}\right) g^{\mu \nu}\left(\partial_{\mu} \phi\right)\left(\partial_{\nu} \phi\right)
\end{aligned}
$$

which indeed shows that the metric on the scalar fields $\varphi_{j}$ is simply $\delta_{i j}$. 


\subsection{Supersymmetric backgrounds}

To find supersymmetric bosonic backgrounds one sets the variations of the spin- $1 / 2$ and spin-3/2 fields to zero. From [6], the gravitational and scalar parts of these variations are:

$$
\begin{aligned}
\delta \psi_{\mu a} & =\mathcal{D}_{\mu} \epsilon_{a}-\frac{1}{6} g W_{a b} \gamma_{\mu} \epsilon^{b} \\
\delta \chi_{a b c} & =\sqrt{2}\left[\gamma^{\mu} P_{\mu a b c d} \epsilon^{d}-\frac{1}{2} g A_{d a b c} \epsilon^{d}\right] .
\end{aligned}
$$

We remind the reader that we use mostly minus metric conventions, and $\left\{\gamma^{\mu}, \gamma^{\nu}\right\}=2 \eta^{\mu \nu}$. For a background with constant scalars, one finds that the vanishing of $\delta \psi$ relates the AdS radius to the eigenvalues of $W_{a b}$. The vanishing of $\delta \chi_{a b c}$ is equivalent, via a tensor identity, to the vanishing of $\delta \psi_{\mu a}$. However, vanishing of $\delta \chi_{a b c}$ directly leads to the condition that one has a supersymmetric background with constant scalars if and only if $\phi=$ 0 and $\partial W / \partial \varphi_{j}=0$ in 3.5. This statement was proved with the help of Mathematica calculations, and is the first of several important places in this work where Mathematica was essential. Note that the conditions $\phi=0$ and $\partial W / \partial \varphi_{j}=0$ are sufficient but not necessary condition for $V$ to have a critical point.

One can easily verify that supersymmetry preserving ground states are obtained by taking:

$$
\varphi_{1}= \pm \frac{1}{2} \log (3), \quad \varphi_{2}=0, \quad \varphi_{3} \equiv \sqrt{6} \alpha=\frac{1}{\sqrt{6}} \log (2), \quad \phi=0 .
$$

All other $S U(2)_{I}$-invariant supersymmetric ground states (besides the trivial $\mathcal{N}=4$ point) are $\mathbb{Z}_{2}$ images of the ones specified in 3.11 , and can be obtained from these either via the interchange $\varphi_{1} \leftrightarrow \varphi_{2}$ or by going to the other component of $O(1,1)$. These supersymmetric ground states were found in [8]. They all have $V_{0}=-\frac{2^{4 / 3}}{3} g^{2}$ and $\mathcal{N}=2$ supersymmetry (in the supergravity sense described at the end of section 1). The supersymmetry generators for 3.11 are given by using the projectors $v_{1}$ and $\tilde{v}_{1}$ of 3.3. If one considers the ground states with $\varphi_{1} \leftrightarrow \varphi_{2}$, then the unbroken supersymmetries are given by $v_{2}$ and $\tilde{v}_{2}$.

In section 6 we will investigate the supergravity spectrum at the critical point specified by 3.11. Our results, and their interpretation within the holographic map between bulk field and boundary operators, will provide detailed evidence that this critical point is the supergravity description of the infrared fixed point of the supersymmetric RG flow described in section 2 . The prima facie evidence for this, as commented in section 2 , is that the value of $V_{0}$ translates into the correct trace anomaly for the supersymmetric 
gauge theory. Before entering into the detailed investigation of the fixed point, we will show how supergravity can provide a description of the entire RG flow from the $\mathcal{N}=4$ UV theory to the $\mathcal{N}=1$ infrared fixed point.

\section{Generalities on holographic RG flows: a c-theorem}

An important aspect of the AdS/CFT correspondence is the notion that the radial coordinate $U$ of AdS can be regarded as a measure of energy. Thus a geometrical cutoff of AdS serves as an ultraviolet regulator. These ideas were implicit in $[1,2,3]$ and have subsequently been developed more fully in $[33,34]$. Phenomena that probe smaller values of $U$ are to be thought of as probing the infrared structure of the theory. For instance, if one computes Green's functions by setting the values of supergravity fields at finite $U$, then making $U$ smaller is believed to provide a natural coarse-graining of the UV details, thereby implementing a version of the Wilsonian renormalization group.

In this context a supergravity "kink" in the $U$-direction interpolating between $U=\infty$ and $U=0$ can be thought of as an explicit construction of a renormalization group flow between a UV fixed point and an IR fixed point of the "boundary" field theory $[20,21]$. Our purpose in this section is to exhibit the general ansatz for a kink and to demonstrate one simple property of kinks that translates via holography into a c-theorem for the boundary theory.

\subsection{The kink ansatz}

We start by considering arbitrary bulk dimension $D$. We are interested in boundary theories that are Poincare invariant in $(D-1)$ dimensions. The most general possible $D$-dimensional bulk metric consistent with this symmetry can be written as

$$
d s^{2}=e^{2 A(r)} \eta_{\mu \nu} d x^{\mu} d x^{\nu}-d r^{2} .
$$

We will always use the mostly minus metric convention, which has the consequence that anti-de Sitter space has a positive cosmological constant. We could generalize 4.1 by replacing $\eta_{\mu \nu}$ by an arbitrary Ricci-flat metric $\gamma_{\mu \nu}$, and none of the analysis in this section or the next would change. If $A(r)=\frac{r}{\ell}$, then 4.1 becomes anti-de Sitter space with $R_{\alpha \beta}=\frac{D-1}{\ell^{2}} g_{\alpha \beta}$. Other standard radial variables for anti-de Sitter space are $U=e^{r / \ell} / \ell[1]$ and $z=x_{0}=\ell e^{-r / \ell}$ 
$[2,3]$. The kink that we shall exhibit in section 5 interpolates between an $r \rightarrow \infty$ "ultraviolet" $A d S_{5}$ region, where $A(r)$ is linear, and an $r \rightarrow-\infty$ "infrared" region, where $A(r)$ is again linear, but with a larger positive slope. A sketch of the spacetime with the spatial boundary dimensions suppressed is shown in Figure 2.

The geometry depicted in Figure 2 is not the only possibility allowed by the ansatz 4.1. For the purpose of studying the AdS/CFT correspondence we are interested in geometries which are asymptotic to anti-de Sitter space on one end: that is, $A(r) \sim \frac{r}{\ell}$ as $r \rightarrow \infty$. Other geometries are possible where curvature singularities appear at finite $r[15,16,35]$. It is also possible that the geometry will have no singularities that are causally connected to the boundary, but that $A(r)$ never recovers linear behavior. The one general restriction, which we will prove in section 4.2 , is $A^{\prime \prime}(r) \leq 0$. This rules out a second anti-de Sitter boundary: it is impossible to have $A^{\prime}(r)$ approach a positive constant as $r \rightarrow \infty$ and a negative constant as $r \rightarrow-\infty$. The monotonicity of $A^{\prime}(r)$ implies a c-theorem, as we shall see.

\subsection{A c-theorem}

The analysis of anomaly coefficients in [31] applies to anti-de Sitter spaces $A d S_{D}$ for any odd bulk dimension $D$. The anomalous value of $\left\langle T_{\mu}^{\mu}\right\rangle$ appears in the supergravity analysis because the cutoff $r=r_{0}$ does not respect diffeomorphism invariance. Different choices of radial variable (including those which mix the coordinates $r$ and $x_{\mu}$ in 4.1) correspond to different but conformally equivalent boundary metrics $\gamma_{\mu \nu}$. The general result of [31] is

$$
\left\langle T_{\mu}^{\mu}\right\rangle=\frac{\text { universal }}{A^{\prime D-2}},
$$

where the universal part is the same for all theories, and is a curvature invariant involving $D-1$ derivatives of the boundary metric. No such invariant exists in odd boundary dimensions. The denominator of 4.2 actually appears in the analysis of [31] as the inverse radius of the anti-de Sitter space, and no particular coordinate choice is implied. Expressing this inverse radius as $A^{\prime}$ is merely a convenience.

The metric

$$
d s^{2}=e^{2 A(r)}\left(\gamma_{\mu \nu} d x^{\mu} d x^{\nu}\right)-d r^{2}
$$

where $\gamma_{\mu \nu}$ is Ricci flat, has a Ricci tensor whose nonvanishing components are

$R_{\mu \nu}=e^{2 A(r)}\left[A^{\prime \prime}+(D-1)\left(A^{\prime}\right)^{2}\right] \gamma_{\mu \nu}, \quad R_{r r}=-(D-1)\left[A^{\prime \prime}+\left(A^{\prime}\right)^{2}\right]$. 
We note that

$$
-(D-2) A^{\prime \prime}=R_{t}^{t}-R_{r}^{r}=G_{t}^{t}-G_{r}^{r}=\kappa_{D}^{2}\left(T_{t}^{t}-T_{r}^{r}\right)
$$
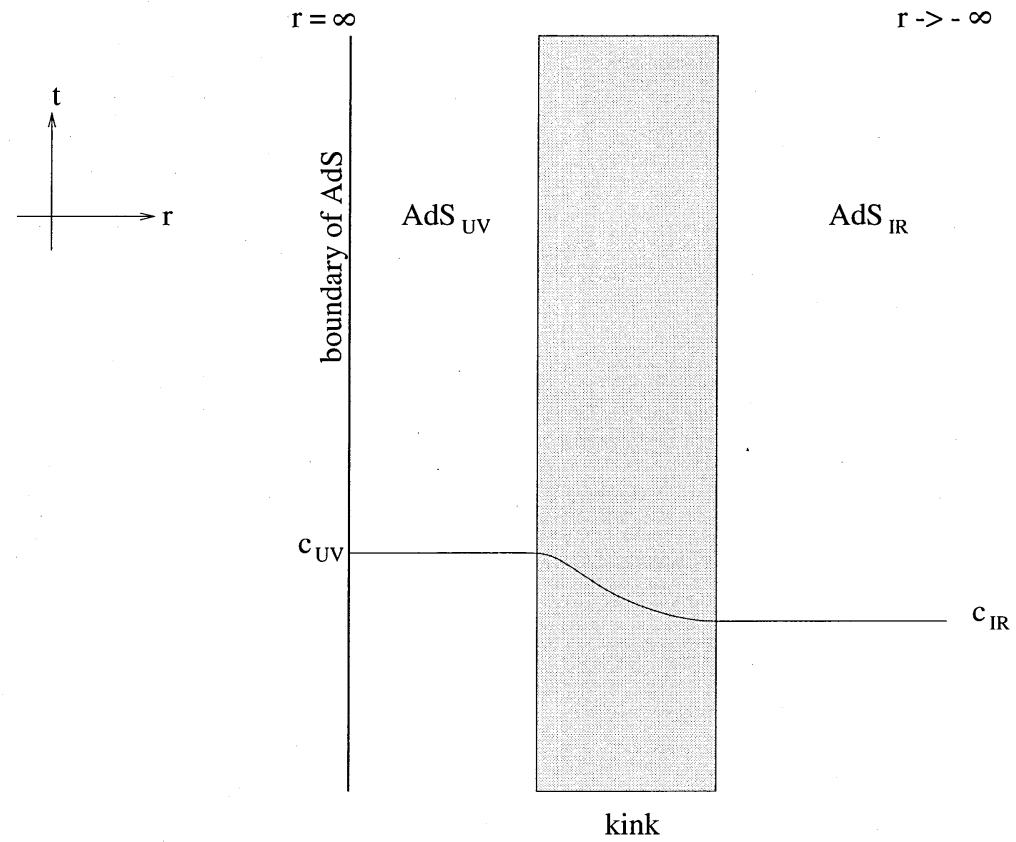

Fig. 2: The supergravity kink interpolates between an asymptotically AdS space in the UV $(r \rightarrow \infty)$ and another in the IR $(r \rightarrow-\infty)$. The value of $\mathcal{C}(r)$ decreases monotonically as one moves toward the infrared, and in the asymptotic regions the value of $\mathcal{C}(r)$ approaches the central charge.

where $\kappa_{D}$ is the $D$-dimensional gravitational constant and in the last equality we have used Einstein's equations, $G_{\alpha \beta}=\kappa_{D}^{2} T_{\alpha \beta}$. Note that we are including any cosmological constant as a term in $T_{\alpha \beta}$ proportional to $g_{\alpha \beta}$. We would now like to argue that the last expression in 4.5 has to be nonnegative: Without resorting to a particular lagrangian, we can try to appeal to some appropriate positive energy condition.

The stress tensor that supports the geometry 4.3 is diagonal:

$$
T_{\beta}^{\alpha}=\operatorname{diag}\left\{\rho,-p_{1},-p_{2}, \ldots,-p_{D-2},-p_{D-1}\right\}
$$

where $p_{D-1}=p_{r}$. There are four different energy conditions in common use: for $D>2$, they read $[36]^{1}$

\footnotetext{
${ }^{1}$ The form we quote for the strong energy condition is the natural one given its motivation in terms of the ricci tensor: $R_{\alpha \beta} \xi^{\alpha} \xi^{\beta} \geq 0$ for timelike or null vectors $\xi^{\alpha}$.
} 


$\begin{array}{lll}\text { Energy condition } & \text { General form } & \text { Diagonal } T_{\beta}^{\alpha} \\ \text { Strong: } & \left(T_{\alpha \beta}-\frac{1}{D-2} g_{\alpha \beta} T\right) \xi^{\mu} \xi^{\nu} \geq 0 & \rho+p_{i} \geq 0, \\ & & (D-3) \rho+\sum_{i} p_{i} \geq 0 \\ \text { Dominant: } & T_{\alpha \beta} \xi^{\alpha} \eta^{\beta} \geq 0 & \rho \geq\left|p_{i}\right| \\ \text { Weak: } & T_{\alpha \beta} \xi^{\alpha} \xi^{\beta} \geq 0 & \rho+p_{i} \geq 0, \rho \geq 0 \\ \text { Weaker: } & T_{\alpha \beta} \zeta^{\alpha} \zeta^{\beta} \geq 0 & \rho+p_{i} \geq 0,\end{array}$

where $\xi^{\alpha}$ and $\eta^{\alpha}$ are arbitrary future-directed timelike or null vectors and $\zeta^{\alpha}$ is an arbitrary null vector. In the second column of 4.7 we have given the general definition of the energy condition, and in the third column we have indicated how it constrains a diagonal stress-energy tensor. What we have called the weaker energy condition is implied by all the others, and what we have called the weak energy condition is implied by the dominant energy condition. Otherwise there are no logical relations among the various conditions. With our convention that any cosmological constant should be included in the stress tensor as a term proportional to $g_{\alpha \beta}$, none of the energy conditions is satisfied by anti-de Sitter space, except the weaker energy condition.

In our case, Poincaré invariance dictates $\rho+p_{i}=0$ for $i=1,2, \ldots, D-2$. We would have $\rho+p_{r}=0$ as well if and only if the cosmological constant is the only contribution to the stress-tensor-and then we recover perfect anti-de Sitter space, so there is no RG flow at all. What we learn, then, is that given Poincaré invariance, the desired monotonicity relation $A^{\prime \prime} \leq 0$ is exactly equivalent to the weaker energy condition. ${ }^{2}$

Consider an example: suppose we had an action

$$
\mathcal{I}_{S G}=\frac{1}{2 \kappa_{D}^{2}} \int d^{D} x \sqrt{|g|}\left[-R+\frac{1}{2} g^{\alpha \beta} \mathcal{M}_{I J} P_{\alpha}^{I} P_{\beta}^{J}-4 V\right]
$$

where $P_{\alpha}^{I}$ represents derivatives of any set of scalar fields, $\mathcal{M}_{I J}$ is some positive definite metric on the scalar manifold, and $V$ is any scalar potential. If we insist on Poincaré invariance, then the scalars can only depend on $r$. Then we find

$$
T_{t}^{t}-T_{r}^{r}=\frac{1}{2 \kappa_{D}^{2}} \mathcal{M}_{I J} P_{r}^{I} P_{r}^{J}
$$

which is positive because $\mathcal{M}_{I J}$ is positive definite. The scalar potential's contribution to $T_{t}^{t}$ and $T_{r}^{r}$ cancels.

Our example is general enough to cover any flow in gauged supergravity involving only the scalar fields and the metric. (It seems difficult to preserve

\footnotetext{
${ }^{2}$ In the initial version of this paper we mistakenly used the dominant energy condition in place of the weaker energy condition. We thank M. Porrati for private communications regarding the relevance of the weaker energy condition which cleared up this error.
} 
Poincaré invariance on the boundary if one turns on any bulk matter fields with spin). But 4.5 , together with the assumption of the weaker energy condition, constitutes an even more general proof that the quantity

$$
\mathcal{C}(r)=\frac{\mathcal{C}_{0}}{A^{D-2}}
$$

is non-increasing along the flow toward the infrared. The parameter, $\mathcal{C}_{0}$, is a constant.

The result 4.2 shows that if the geometry is anti-de Sitter, then the anomaly coefficients of the corresponding conformal field theory are proportional to $\mathcal{C}$. With appropriate definitions of those anomaly coefficients and of $\mathcal{C}_{0}$, we can say that $\mathcal{C}$ coincides with the anomaly coefficients for anti-de Sitter geometries. In the duality of $\mathcal{N}=4 S U(N)$ super-Yang-Mills theory with $\mathcal{N}=8$ gauged supergravity, one can show that

$$
\mathcal{C}(r)=\frac{g^{3} N^{2} / 32}{A^{\prime}(r)^{3}}
$$

is the appropriate normalization to give $\mathcal{C}=a=c=\frac{N^{2}}{4}$ for the unperturbed maximally supersymmetric anti-de Sitter vacuum of the supergravity theory, and $\mathcal{C}=a=c=\frac{27}{128} N^{2}$ for the one-quarter supersymmetric vacuum found in [8]. In 4.11, $g$ is the gauge coupling in the five-dimensional supergravity, and it has units of energy. The important thing to realize is that once we have fixed $\mathcal{C}_{0}$ within a particular supergravity theory, the formula 4.10 gives a uniform prescription for computing the anomaly coefficients in any conformal theory dual to an anti-de Sitter vacuum of that supergravity theory.

Now we want to consider a kink spacetime which is asymptotic to anti-de Sitter spaces for $r \rightarrow \pm \infty$. The analysis of [31] does not apply directly to such a spacetime. But its asymptotic limits as $r \rightarrow \pm \infty$ are anti-de Sitter vacua, and we can think of obtaining the UV and IR anomaly coefficients for these vacua from 4.10. To apply the analysis of [31] directly we must "lift out" one asymptotically anti-de Sitter end from the kink and make it into an exactly anti-de Sitter spacetime. But the answer for $\mathcal{C}$ is exactly the same as if we had evaluated $\lim _{r \rightarrow \pm \infty} \mathcal{C}(r)$ in the original non-anti-de Sitter kink geometry. This is precisely analogous to the situation in field theory: properties pertaining narrowly to a given fixed point do not depend on a particular flow that leads into it or out of it; such properties can be studied just as well by considering the trivial flow which remains forever at the fixed point.

It is difficult or impossible to give a scheme-independent definition of the central charge away from conformal fixed points. Flows may exist which 
pass arbitrarily close to one fixed point on their way to another. For these a "scaling region" exists where $A^{\prime}$ is much larger than higher derivatives of $A$. The function, $\mathcal{C}(r)$, in such a region is close to the anomaly coefficient of the nearby fixed point.

In [37], the monotonicity of the c-function followed trivially from the fact that the RG flow was the gradient flow of the c-function. In general it seems difficult to prove any analog of that statement in four dimensions. However, for supersymmetric supergravity kinks, an analogous statement can be proven: as we will explain in section $5, A^{\prime}$ can be expressed as a function of the scalar fields (without derivatives), and the trajectory of the scalars throughout the flow is specified by gradient flow of that function.

Our proof of a holographic c-theorem also applies to flows such as the one envisaged in [17], where light string states from twisted sectors are involved. In addition, it would apply to flows involving any or all of the massive Kaluza-Klein states arising from any compactification of ten-dimensional supergravity down to five-dimensions. It even applies if the matter lagrangian includes higher derivative terms, provided the weaker energy condition continues to hold. However, the proof does rely on Einstein's equations. It would be interesting to explore whether the argument can be extended to cover the alterations in the gravitational action that arise through $\alpha^{\prime}$ corrections. We should also add that the inequality $A^{\prime \prime} \leq 0$ turned out to be exactly the weaker energy condition on the stress-tensor supporting the geometry, so if consistent matter violating the weaker energy condition exists in the bulk in a regime where the classical gravity approximation is valid, it seems likely that one could use it to construct holographically a boundary theory which violates the c-theorem.

In summary, we seem to have a dimension-independent proof of a ctheorem from the weaker energy condition. It applies to theories where a gravity dual can be found in which Einstein's equations hold, and in which the gravity approximation applies. Besides the obvious large $N$, strong coupling restrictions, this also amounts in four dimensions to the restriction $a-c=0$, at least to leading order in large $N$. (The $1 / N$ corrections were explored in $[38,39]$.) The proof of the theorem, equation 4.5 , is appropriately trivial for such a general truth.

There have been other recent efforts toward a c-theorem in higher dimensions: $[40,41]$ take a field theoretic approach, [42] works with thermodynamic quantities, ${ }^{3}$ and [43] uses the AdS/CFT correspondence. When this work

\footnotetext{
${ }^{3}$ Monotonicity of the thermodynamic c-function considered in [42] follows from the inequality $\left\langle T_{\mu}^{\mu}\right\rangle \geq 0$, where \langle\rangle indicates a thermal average and $T_{\mu \nu}$ is the stress tensor in
} 
was complete, we learned that an equivalent form of the c-function in 4.11 was discussed in the later versions of [20]. ${ }^{4}$ Its monotonicity was checked for the flows considered there.

\section{Supersymmetric flows}

To make the correspondence between the supergravity theory and the boundary theory on the brane more complete, one should be able to find the flow between the central critical point with maximal supersymmetry and the $\mathcal{N}=2$ supersymmetric critical points described in section 3.2 . In particular one should be able to preserve $\mathcal{N}=1$ supersymmetry on the branes all along the flow, i.e., the kink should itself be supersymmetric. Thus we once again study the Killing spinor conditions, i.e. the vanishing of 3.10, but the metric now takes the form 4.1 and the scalars depend on $r$. The vanishing of $\delta \chi_{a b c}$ directly relates $r$ derivatives of the scalars to the gradient of $W$. Specifically, to solve $\delta \chi_{a b c}=0$ we find that we must first set $\phi=0$. There are then two classes of supersymmetry preserving kink solutions that are related by $\varphi_{1} \leftrightarrow \varphi_{2}$. Here we will describe the family that contains the flow to 3.11. We will use the same $\gamma$-matrix conventions as [6].

For an unbroken supersymmetry along the flow of the form

$$
\epsilon^{a}=\left(v_{1}^{a}+\tilde{v}_{1}^{a} \gamma^{r}\right) e^{A(r) / 2} \eta
$$

where $v_{1}^{a}$ and $\tilde{v}_{1}^{a}$ are given in 3.3 and $\eta$ is a constant spinor, the vanishing of $\delta \chi_{a b c}$ requires:

$$
\frac{d \varphi_{j}}{d r}=\frac{g}{2} \frac{\partial W}{\partial \varphi_{j}},
$$

where $W=\left.\lambda_{1}\right|_{\phi=0}$ (see 3.4). In this section, except as indicated explicitly, the subscript $j$ on $\varphi_{j}$ runs from 1 to 3 . One can show that for such an $\epsilon^{a}$ to satisfy the symplectic-Majorana condition in five dimensions, $\eta$ must be a Majorana spinor in the four dimensions of the brane. To verify this one uses: $\Omega^{a b} v_{1}^{b}=-\tilde{v}_{1}^{a}, \Omega^{a b} \tilde{v}_{1}^{b}=v_{1}^{a}$, where $\Omega$ is the $U S p(8)$ symplectic form. One also needs the fact that the four-dimensional charge conjugation matrix, $\hat{C}$, may be written as $\hat{C}=\gamma^{r} C$, where $C$ is the five-dimensional charge conjugation matrix. As a result, we see that the kink preserves a single, four-dimensional Majorana supersymmetry, i.e. the flow preserves $\mathcal{N}=1$ supersymmetry on the brane. Mathematica was again essential to deduce the simple result 5.2 from the 48 conditions $\delta \chi_{a b c}=0$.

what we would call the boundary theory.

${ }^{4}$ We thank A. Zaffaroni for bringing this work to our attention, and also M. Porrati for subsequent discussions. 
The vanishing of $v_{1}^{a} \delta \psi_{\mu a}$ and $\tilde{v}_{1}^{a} \delta \psi_{\mu a}$ yields one more equation:

$$
A^{\prime}=-\frac{g}{3} W \text {. }
$$

One can easily verify that a solution to the first order equations 5.2 and 5.3 automatically satisfies both the gravitational and scalar equations of motion arising from 3.6, 3.9 and 3.5.It is worth noting that the equations 5.2, 5.3 have a certain similarity to the attractor mechanism [66]. There is also some relation to the supergravity domain wall literature (see for example [67]).

The other family of supersymmetric kinks is obtained by taking $W=$ $\left.\lambda_{2}\right|_{\phi=0}$, and replacing $v_{1}, \tilde{v}_{1}$ by $v_{2}, \tilde{v}_{2}$. The conclusions are otherwise identical.

As promised in section 4.2, we have produced a function of the scalars, $W$, which is related to the c-function by

$$
\mathcal{C}(r)=\frac{27}{32} \frac{N^{2}}{|W|^{3}}
$$

and whose gradient flow trajectories determine the scalar profiles in the supergravity kink. Since the scalars are viewed in the AdS/CFT correspondence essentially as parameters in the lagrangian, we can assert that the supersymmetric renormalization group flow is gradient flow. The monotonicity of $A^{\prime}$ is of course a trivial consequence: using 5.3 and 5.2 one obtains

$$
A^{\prime \prime}=-\frac{g^{2}}{6} \sum_{j}\left|\frac{\partial W}{\partial \varphi_{j}}\right|^{2}=-\frac{2}{3} \sum_{j}\left|\frac{\partial \varphi_{j}}{\partial r}\right|^{2} \leq 0 .
$$

The monotonicity of $A^{\prime}$ is related to the local potential energy of the superkink. Perhaps more fundamentally, recall that the value of the superpotential at either end of a kink may be thought of as determining the topological sector [44]. ${ }^{1}$ Thus the change in $\mathcal{C}(r)$ as $r$ goes from large positive values (the ultraviolet) to large negative values (the infrared) is a measure of the topological charge of the superkink.

Note there are infinitely many supersymmetry preserving solutions starting at the point of maximal supersymmetry, $\varphi_{j}=0, \phi=0$. Most of these

\footnotetext{
${ }^{1}$ Unlike standard kinks, where a field flows from one minimum of a potential to another, our kink flows from a local maximum of the gauged supergravity potential to a saddle point. As long as there are relevant perturbations in the gauge theory, there will always be directions in the potential where the second derivative is negative, but that does not spoil stability of the saddle point vacuum in supergravity provided the BreitenlohnerFreedman bound is satisfied. Instead of using finite total energy to restrict the asymptotic behavior of the kink, we can use the requirement that there be no naked singularities. With this criterion our kink is locally unique.
} 
solutions go to $W \rightarrow-\infty$, and it requires the correct initial conditions to reach the non-trivial supersymmetric critical point. We can set $\phi=0$ and $\varphi_{2}=0$ throughout the flow: the first restriction is necessary to preserve supersymmetry, and the second is necessary to preserve the $U(1)$ symmetry which is present at the infrared fixed point (as commented in section 2, a nonanomalous $U(1)$ current exists for the entire field RG flow, and it should be reflected in a $U(1)$ symmetry of the supergravity solution). One can check that $\left.\frac{\partial W}{\partial \varphi_{2}}\right|_{\varphi_{2}=0}$ vanishes, so $\varphi_{2}=0$ is also consistent with supersymmetry. The superpotential now reduces to

$$
W=\frac{1}{4 \rho^{2}}\left[\cosh \left(2 \varphi_{1}\right)\left(\rho^{6}-2\right)-\left(3 \rho^{6}+2\right)\right] .
$$

The contour maps of $V$ and $W$ on the $\left(\alpha=\frac{1}{\sqrt{6}} \varphi_{3}, \varphi_{1}\right)$ parameter space are shown in Figure 3. The map of $V$ shows five extrema. Point 1 is the maximally supersymmetric point. It is a local maximum of both $V$ and $W$. Points 2 and 3 are $\mathbb{Z}_{2}$ equivalent $S U(3)$ invariant points. In the $\varphi_{1}$ direction these points sit at a quadratic minimum of $V$; in the $\varphi_{3}$ direction they sit on a cubic inflection point. Points 4 and 5 are $\mathbb{Z}_{2}$ equivalent $\mathcal{N}=2$ supersymmetric points. These points are generic quadratic saddle points of both $V$ and $W$, and the contours surrounding them are locally hyperbolas. The superkink which we study follows a path from the central maximum of $W$ down the "ridge" to the saddle point labelled 4 . We have been unable to find an analytic solution to the steepest descent equations, but we will discuss a numerical solution as well as analytic treatments of the endpoints at the end of this section.
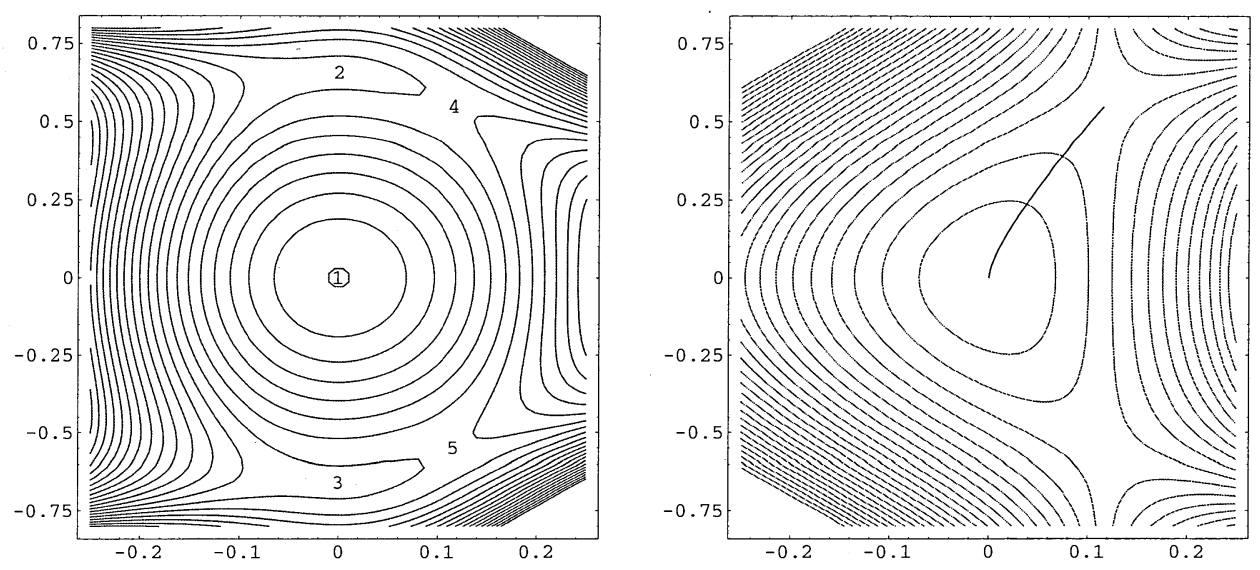

Fig. 3: The contour map of $V$ (on the left) and $W$ (on the right), with $\varphi_{1}$ on the vertical axis and $\alpha=\frac{1}{\sqrt{6}} \varphi_{3}$ on the horizontal axis. The five labelled points are the only extrema of $V$ in this plane. $V$ has vanishing first derivatives in all directions orthogonal to the plane. A numerical solution of the steepest descent equations is 
shown superimposed on the contour plot of $W$.

Consider the mass matrix, $M_{i j}$, for the $\varphi_{j}$ at a critical point of $W$. Differentiating 3.5 , setting $\phi=0$ and using $\partial W / \partial \varphi_{j}=0$, one gets:

$$
\begin{aligned}
M_{i j} & =\frac{\partial^{2} V}{\partial \varphi_{i} \partial \varphi_{j}}=\frac{g^{2}}{4} \sum_{k=1}^{3} \frac{\partial^{2} W}{\partial \varphi_{i} \partial \varphi_{k}} \frac{\partial^{2} W}{\partial \varphi_{k} \partial \varphi_{j}}-\frac{2 g^{2}}{3} W \frac{\partial^{2} W}{\partial \varphi_{i} \partial \varphi_{j}} \\
& =\left(\frac{g W}{3}\right)^{2} \mathcal{U}_{i k}\left(\mathcal{U}_{k j}-4 \delta_{k j}\right), \text { where } \mathcal{U}_{i j} \equiv\left(\frac{3}{2 W}\right) \frac{\partial^{2} W}{\partial \varphi_{i} \partial \varphi_{j}}
\end{aligned}
$$

The mass scale is set by the inverse radius, $1 / \ell$, of the AdS space, and this may be written $1 / \ell=\sqrt{-V / 3}=-\frac{g}{3} W$, where we have used 3.5. For $\phi=0$ the matrix $\mathcal{U}_{i j}$ is real and symmetric, and so has real eigenvalues, $\delta_{k}$, $k=1,2,3$. It follows that the eigenvalues of $\ell^{2} M_{i j}^{2}$ are simply:

$$
m_{k}^{2}=\delta_{k}\left(\delta_{k}-4\right) \text {. }
$$

Combining this with the results of $[2,3]$ we see that a particular eigenvalue $\delta_{k}$ of $\left(\frac{3}{2 W}\right) \frac{\partial^{2} W}{\partial \varphi_{i} \partial \varphi_{j}}$ is related to the conformal dimension $\Delta_{k}$ of the field theory operator dual to the variation of the fields $\varphi_{j}$ in the corresponding eigendirection either by $\Delta_{k}=\delta_{k}$ or $\Delta_{k}=4-\delta_{k}$.

Since $U=e^{A(r)}$ is the renormalization scale on the flow, we should be able to read off the leading contributions to the $\beta$-functions of the couplings $\varphi_{j}$ in the neighborhood of the end points of the flow. Note that $\frac{d}{d r}=A^{\prime} U \frac{d}{d U}=$ $-\frac{g}{3} W U \frac{d}{d U}$. Hence, 5.2 becomes

$$
U \frac{d}{d U} \varphi_{j}=-\frac{3}{2} \frac{1}{W} \frac{\partial W}{\partial \varphi_{j}} \approx-\left.\mathcal{U}_{j k}\right|_{\text {crit.pt. }} \delta \varphi_{k},
$$

where we have expanded to first order in the neighborhood of a critical point, and where $\mathcal{U}_{j k}$ is defined in 5.7. ${ }^{2}$ The eigenvalues, $\delta_{j}$, of $\mathcal{U}_{j k}$ thus determine the behavior of the scalars $\varphi_{j}$ near the critical points. First, to depart the UV fixed point $(U=+\infty)$ the flow must take place in directions in which the eigenvalues are positive (i.e. the corresponding operators must be relevant), and to approach the IR fixed point $(U \rightarrow 0)$ the eigenvalues must be negative

\footnotetext{
${ }^{2}$ We regard $U=e^{A(r)}$ as a natural measure of energy scale because of the conformal factor $e^{2 A(r)}$ in the metric 4.1. Other definitions have been proposed [34]. For instance, $U$ could be taken as the inverse of the coordinate time $t$ it takes for a light ray to propagate to a given radius. This gives $U=\left(\int_{r}^{\infty} d \tilde{r} e^{-A(\tilde{r})}\right)^{-1}$. The difference is immaterial in 5.9 because we are operating close to the UV fixed point.
} 
(i.e. the corresponding operators must be irrelevant). At the central critical point, the eigenvalues are $\delta_{k}=1,2,3$, corresponding to $\varphi_{1}, \varphi_{3}, \varphi_{2}$. Since $\varphi_{2} \equiv 0$ on this flow, only the eigenspaces of eigenvalue 1 and 2 are used. The supergravity fields $\varphi_{1}$ and $\varphi_{3}$ are dual to operators of dimension 3 and 2 , respectively: the fermion and scalar mass terms.

Indeed, one can perform the analysis of the ridge-line flow in the neighborhood of $\varphi_{i}=0$ by considering the series expansion

$$
W=-\frac{3}{2}-\frac{\varphi_{1}^{2}}{2}-\varphi_{3}^{2}+\sqrt{\frac{8}{3}} \varphi_{1}^{2} \varphi_{3}+\ldots
$$

where we have set $\varphi_{2}=0$. We will operate in units where $g=2$. Keeping only the quadratic terms in the expansion leads to $\varphi_{1} \sim \pm e^{-r}, \varphi_{3} \sim \pm e^{-2 r}$, and with this behavior the cubic term is of the same order as the quadratic terms. We must keep this term even in a lowest-order analysis of the behavior of $\varphi_{3}$. The correct analysis of the asymptotics is

$$
\left.\begin{array}{rlrl}
{\left[\frac{d}{d r}+1\right] \varphi_{1}} & =0 & \varphi_{1} & \sim a_{0} e^{-r} \\
{\left[\frac{d}{d r}+2\right] \varphi_{3}} & =\sqrt{\frac{8}{3}} \varphi_{1}^{2} & \varphi_{3} & \sim \sqrt{\frac{8}{3}} a_{0}^{2} r e^{-2 r}+a_{1} e^{-2 r}
\end{array}\right\} \quad \text { as } r \rightarrow \infty .
$$

It is straightforward to verify that higher order terms in 5.10 do not change this story: roughly speaking, two powers of $\varphi_{1}$ are worth one power of $\varphi_{3}$ in the expansion of $W$. The integration constant $a_{0}$ is, up to factors 'of order unity, the fermion mass resulting from the term $\frac{1}{2} m \operatorname{Tr} \Phi_{3}^{2}$ in the superpotential (see 2.1). Supersymmetry fixes the value of the coefficient of $r e^{-2 r}$ in the asymptotics of $\varphi_{3}$. This coefficient, again up to factors of order unity, is the square of the boson mass resulting from the mass term in the superpotential. The integration constant $a_{1}$ is not determined by supersymmetry, and reflects the possibility of different states in the theory, only one of which is the vacuum which preserves conformal invariance. We do not yet have a way of determining $a_{1}$ analytically, but we will shortly discuss its numerical evaluation.

The flow near the IR critical point 3.11 is a little simpler. The eigenvalues, $\delta_{k}$, of $\mathcal{U}_{i j}$ are $1+\sqrt{7}, 1-\sqrt{7}, 3$. The only negative one is $1-\sqrt{7}$, and so the corresponding eigenvector determines the direction from which the flow approaches the fixed point. The asymptotics (again in units where $g=2$ ) is 


$$
\left.\begin{array}{c}
\varphi_{1} \sim \frac{1}{2} \log 3-b_{0} e^{\lambda r} \\
\varphi_{3} \sim \frac{1}{\sqrt{6}} \log 2-\frac{\sqrt{7}-1}{\sqrt{6}} b_{0} e^{\lambda r}
\end{array}\right\} \quad \text { as } r \rightarrow-\infty .
$$

The irrelevant operator in the field theory that controls this flow has dimension $3+\sqrt{7}$. Because of the possibility of shifting $r$ additively, the coefficients $a_{0}, a_{1}$, and $b_{0}$ are not all independently meaningful. The two combinations that are invariant are $b_{0} a_{0}^{\lambda}$ and $\frac{a_{1}}{a_{0}^{2}}+\sqrt{\frac{8}{3}} \log a_{0}$. Additive shifts of $r$ amount to rigid dilations of the boundary, and the flow is invariant under these if we make a corresponding shift of the parameter $m$ in the superpotential.

A numerical solution interpolating between 5.11 and 5.12 is easier to find if one starts from the infrared end. Then the differential equations are stable in that a small perturbation in the initial conditions damps out over time. The opposite is true of the flow from the ultraviolet end to the infrared end, which is appropriate since relevant deformations become more important in the infrared. Our results for the invariant combinations of coefficients are

$$
b_{0} a_{0}^{\lambda} \approx 0.1493 \quad \frac{a_{1}}{a_{0}^{2}}+\sqrt{\frac{8}{3}} \log a_{0} \approx-1.4694 .
$$

Minus the logarithm of the first number can be thought of roughly as the width of the kink. The second number represents the choice of vacuum state that leads to infrared physics with non-vanishing central charge.

\section{Supergravity fields and the operator map}

The symmetries of the supersymmetric critical point [8] in $A d S_{5}$ include $\mathcal{N}=2$ supersymmetry and the gauge symmetry $S U(2)_{I}$, so the supergravity fields should be classified in representations of the corresponding superalgebra $S U(2,2 \mid 1) \times S U(2)_{I}$. The AdS/CFT correspondence requires a one to one map between these fields and gauge invariant composite operators of the boundary theory, in this case the mass deformed $\mathcal{N}=4$ super-Yang-Mills theory discussed in section 2 . In $\mathcal{N}=1$ superspace this theory contains a chiral spinor superfield $W_{\alpha}$ describing the gauge multiplet, and three chiral scalar superfields $\Phi_{i}, i=1,2,3$, describing supermatter. The component expansions are

$$
W_{\alpha}=\lambda_{\alpha}+\theta^{\beta}\left[\frac{1}{2}\left(\sigma^{\mu} \bar{\sigma}^{\nu}\right)_{\beta \alpha} F_{\mu \nu}+i \epsilon_{\alpha \beta} D\right]-\theta^{2} i \sigma_{\alpha \dot{\beta}}^{\mu} D_{\mu} \bar{\lambda}^{\dot{\beta}}
$$




$$
\Phi_{i}=z_{i}+\theta^{\alpha} \psi_{\alpha i}+\theta^{2} F_{i}
$$

In this section we will discuss the spectrum of fields in bulk supergravity theory and propose a map to operators of the boundary theory. Some aspects of the corresponding unitary representations of $S U(2,2 \mid 1)[45,46]$ are summarized in Appendix B with a brief discussion at the end of this section.

The main technical task is to study the action of the gauged supergravity theory of $[6,7]$ expanded to quadratic order about the $\mathcal{N}=2$ critical point and to compute the mass eigenvalues of all fields. This is an unfortunately complex matter. The general approach is discussed in Appendix A. We will give the decompositions of supergravity fields - which transform under $U S p(8) \times S U(4)$ in the full theory - with respect to the unbroken gauge subgroup $S U(2)_{I} \times U(1)_{R}$ at the critical point. This will help readers understand the group-theoretic structure of the results. The detailed masses, however, depend crucially upon accurate calculation of the scalar 27-bein $\mathcal{V}_{a b}{ }^{A B}$ at the critical point and of the $U S p(8)$ tensors $W_{a b}$ and $A_{a b c d}$, which are given by a quadratic and a quartic expression, respectively, in terms of that 27-bein. Moreover, the computation of the masses of scalar fields requires quadratic expansions of those tensors about the critical point, and a similarly complicated determination of the kinetic term.

\subsection{The fermion masses}

The relevant part of the supergravity action reads $[6]:^{1}$

$$
\begin{aligned}
e^{-1} L_{F}= & -\frac{1}{2} i \bar{\psi}_{\mu}^{a} \gamma^{\mu \nu \rho} D_{\nu} \psi_{\rho a}+\frac{1}{12} i \bar{\chi}^{a b c} \gamma^{\mu} D_{\mu} \chi_{a b c}-\frac{1}{4} i g W_{a b} \bar{\psi}_{\mu}^{a} \gamma^{\mu \nu} \psi_{\nu}^{b} \\
& +\frac{1}{6} \sqrt{\frac{1}{2}} i g A_{d a b c} \bar{\chi}^{a b c} \gamma^{\mu} \psi_{\mu}^{d}+\frac{1}{2} i g \bar{\chi}^{a b c}\left(\frac{1}{2} A_{b c d e}-\frac{1}{12} \Omega_{b d} W_{c e}\right) \chi_{a}{ }^{d e}
\end{aligned}
$$

where the spin-3/2 field $\psi_{\mu}^{a}$ and the spin- $1 / 2$ field $\chi^{a b c}$ transform as 8 and 48 of $U S p(8)$, respectively, and satisfy the symplectic Majorana conditions.

There is a super-Higgs effect in this sector. The simplest way to treat it is to observe that the spin- $1 / 2$ supersymmetry transformation rule in [6] reduces to, cf. 3.10 ,

$$
\delta \chi_{a b c}=-\sqrt{\frac{1}{2}} g A_{d a b c} \epsilon^{d}
$$

and this describes a rank 6 linear transformation from the 8-dimensional space of the $\epsilon^{a}$ into the 48-dimensional space of the $\chi_{a b c}$. Thus one can fix

\footnotetext{
${ }^{1}$ In this section, to conform with the notation in [6] we use indices $\mu, \nu$ for space-time indices in five dimensions.
} 
the gauge by using 6 spinor parameters of the broken supersymmetries to eliminate the 6 fields in the range of the transformation. Those are precisely the same 6 fields as in the mass-mixing term in the Lagrangian 6.2 which can thus be dropped in this gauge. The action on the space of remaining fields has no mixing between the spin- $3 / 2$ and spin- $1 / 2$ fields and it is clear that the remaining spin-3/2 part of the Lagrangian is not modified in the process.

The final diagonalization of the spin-3/2 action can be described quite explicitly. Since the kinetic term is already in the canonical form, all that remains is to diagonalize the symmetric matrix $W_{a b}$, which at the critical point is given in (A.42). It has two 2-dimensional eigenspaces and one 4dimensional eigenspace as follows

$$
\begin{aligned}
& \lambda_{1}=-2^{2 / 3}, \quad v_{1}=(1,0,0,0,0,1,0,0), \quad \tilde{v}_{1}=(0,1,0,0,-1,0,0,0), \\
& \begin{aligned}
& = \\
\lambda_{2} & =-\frac{4}{3} 2^{2 / 3}, \quad v_{2}=(0,0,0,1,0,0,1,0), \quad \tilde{v}_{2}=(0,0,1,0,0,0,0,-1),
\end{aligned}
\end{aligned}
$$

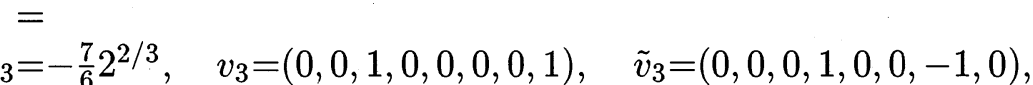

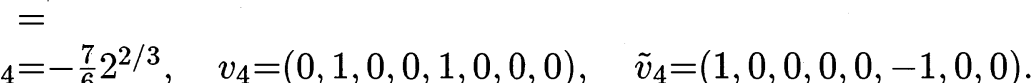

Define projections $\psi_{\mu, i}^{1}=\psi_{\mu}^{a} v_{i a}, \psi_{\mu, i}^{2}=\psi_{\mu}^{a} \tilde{v}_{i a}$ of $\psi_{\mu}^{a}$ onto the eigenspace $\left(v_{i}, \tilde{v}_{i}\right)$. In terms of those modes we get a standard kinetic term, with the symplectic matrix $\omega$ acting by $\omega v_{i}=\tilde{v}_{i}, \omega \tilde{v}_{i}=-v_{i}$, and a standard symplectic mass term [47]. The quadratic action in each eigenspace reads

$$
e^{-1} L_{3 / 2}=\frac{1}{2} \omega_{\alpha \beta} \bar{\psi}_{\mu, i}^{\alpha} \gamma^{\mu \nu \rho} D_{\nu} \psi_{\rho, i}^{\beta}+\frac{1}{2} \frac{m_{i}}{\ell} \delta_{\alpha \beta} \bar{\psi}_{\mu, i}^{\alpha} \gamma^{\mu \nu} \psi_{\nu, i}^{\beta}
$$

where

$$
\frac{m_{i}}{\ell}=\frac{3}{2} g \lambda_{i}, \quad \frac{1}{\ell}=-\frac{1}{3} g W=\frac{2^{2 / 3}}{3} g .
$$

As expected the sector with $m_{1}=(3 / 2) g \ell \lambda_{1}$ is the gravitino of the $\mathcal{N}=2$ theory and the remaining states are massive spin- $3 / 2$ fields with masses $m_{i}, i=2,3,4$, given in 6.5. This is consistent with the decomposition $U S p(8) \rightarrow S U(2)_{I} \times U(1)_{R}$

$$
8 \rightarrow\left(\mathbf{2}_{1 / 2} \oplus \mathbf{2}_{-1 / 2}\right) \oplus\left(\mathbf{1}_{1} \oplus \mathbf{1}_{-1}\right) \oplus 2 \times \mathbf{1}_{0} .
$$

of which the first two singlets, $\mathbf{1}_{1} \oplus \mathbf{1}_{-1}$, correspond to the gravitino in $\mathcal{N}=2$ theory. 
The diagonalization of the action for the remaining 42 spin- $1 / 2$ fields is more involved and we have carried it out in Mathematica using formulae from Appendix A. As with spin-3/2, the symplectic Majorana condition requires that the masses occur in pairs $\pm m$. We have checked that the mass eigenstates transform correctly under $S U(2)_{I} \times U(1)_{R}$ as predicted by the following branching rule

$$
\begin{aligned}
48 \rightarrow & \left(\mathbf{3}_{1} \oplus \mathbf{3}_{-1}\right) \oplus 2 \times \mathbf{3}_{0} \oplus 2 \times\left(\mathbf{2}_{3 / 2} \oplus \mathbf{2}_{-3 / 2}\right) \\
& \oplus 4 \times\left(\mathbf{2}_{1 / 2} \oplus \mathbf{2}_{-1 / 2}\right) \oplus\left(\mathbf{1}_{2} \oplus \mathbf{1}_{-2}\right) \\
& \oplus 3 \times\left(\mathbf{1}_{1} \oplus \mathbf{1}_{-1}\right) \oplus 4 \times \mathbf{1}_{0} .
\end{aligned}
$$

The six Goldstino modes that are absorbed into massive spin- $3 / 2$ fields are identified using 6.6 as $\left(\mathbf{2}_{1 / 2} \oplus \mathbf{2}_{-1 / 2}\right) \oplus 2 \times \mathbf{1}_{0}$.

\subsection{The boson masses}

The computation of the masses of the vector fields, $A_{\mu}^{I J}$, and the antisymmetric tensor fields, $B_{\mu \nu}^{I \alpha}$, is quite straightforward and similar to the one above for the fermions. One must consider two terms in the action

$$
e^{-1} L_{A B}=-\frac{1}{8} H_{\mu \nu a b} H^{\mu \nu a b}+\frac{1}{8 g e} \epsilon^{\mu \nu \rho \sigma \tau} \epsilon_{\alpha \beta} \delta_{I J} B_{\mu \nu}^{I \alpha} D_{\rho} B_{\sigma \tau}{ }^{J \beta}
$$

where

$$
H_{\mu \nu}^{a b}=F_{\mu \nu I J} \mathcal{V}^{I J a b}+B_{\mu \nu}^{I \alpha} \mathcal{V}_{I \alpha}^{a b}
$$

and $F_{\mu \nu I J}$ are the field strengths for the vector fields. Note that the first term in 6.8 gives rise to the kinetic term for the vectors, but the mass term for the antisymmetric tensors, ${ }^{1}$ and is the only source of cross-terms. At the quadratic level the Chern-Simons like kinetic term for the antisymmetric tensors is invariant under a shift

$$
B_{\mu \nu}^{I \alpha} \rightarrow B_{\mu \nu}^{I \alpha}+X_{J K}^{I \alpha} F_{\mu \nu}^{J K},
$$

in which $X^{I \alpha}{ }_{J K}$ is chosen to remove the crosis terms. In this process the kinetic term and thus the mass eigenvalues of the vector fields are modified.

The decomposition of the vector fields with respect to $S U(4) \supset S U(2)_{I} \times$ $U(1)_{R}$ is

$$
15 \quad \rightarrow \quad \mathbf{3}_{0} \oplus \mathbf{1}_{0} \oplus\left(\mathbf{2}_{3 / 2} \oplus \mathbf{2}_{-3 / 2}\right) \oplus\left(\mathbf{2}_{1 / 2} \oplus \mathbf{2}_{-1 / 2}\right) \oplus\left(\mathbf{1}_{1} \oplus \mathbf{1}_{-1}\right) \oplus \mathbf{1}_{0} .
$$

\footnotetext{
${ }^{1}$ This observation may be rather puzzling at the first sight - it is, however, quite natural since the antisymmetric tensors correspond to field strengths of vector fields in the ungauged theory $[5,6,7]$.
} 
One expects that all but the first two modes, corresponding to unbroken gauge symmetries, become massive. As we have discussed above, the actual values of the masses are effected by the mixing with the antisymmetric tensor fields. However, the usual Higgs effect, in which 11 massless Goldstone bosons are eaten by the massive vector fields, does not directly affect the calculation. Therefore the masses can be determined from 6.8 once the proper shift 6.10 has been made.

Since the antisymmetric tensor fields have a first order action, their masses come in $\pm m$ pairs corresponding to the terms in the $S U(2)_{I} \times U(1)_{R}$ decomposition

$$
12 \rightarrow 2 \times\left(\mathbf{2}_{1 / 2} \oplus \mathbf{2}_{-1 / 2}\right) \oplus 2 \times\left(\mathbf{1}_{1} \oplus \mathbf{1}_{-1}\right) .
$$

As for the vectors, the main difficulty here is to compute the metric $(\mathcal{V V})_{I \alpha J \beta}$ that encodes the dependence of the masses on the expectation values of the scalar fields.

The calculation of masses of the scalar fields presents additional difficulty if one wants to consider the full set of 42 fields, rather than the restricted subset corresponding to the gauge fixed subspace of the scalar manifold $\mathcal{S}$ discussed in section 3 . In the general case one must calculate carefully both the $\sigma$-model kinetic action at the critical point and expand the potential to the quadratic order in all directions. The calculation can be simplified by restricting to different sectors of $S U(2)_{I} \times U(1)_{R}$, one at a time, which in this case are given by the branching

$$
\begin{aligned}
\mathbf{4 2} \rightarrow \quad & \mathbf{3}_{0} \oplus 2 \times\left(\mathbf{3}_{1} \oplus \mathbf{3}_{-1}\right) \oplus\left(\mathbf{2}_{3 / 2} \oplus \mathbf{2}_{-3 / 2}\right) \\
& \oplus 3 \times\left(\mathbf{2}_{1 / 2} \oplus \mathbf{2}_{-1 / 2}\right) \oplus 2 \times\left(\mathbf{1}_{2} \oplus \mathbf{1}_{-2}\right) \oplus\left(\mathbf{1}_{1} \oplus \mathbf{1}_{-1}\right) \\
& \oplus 5 \times \mathbf{1}_{0} .
\end{aligned}
$$

By carrying out a careful diagonalization we find a perfect agreement of the mass spectrum with this decomposition. In particular, the result displays 13 massless states of which the two real $S U(2)_{I}$ singlets are the dilaton and the axion and the remaining 11 are the Goldstone bosons from the breaking $S U(4) \rightarrow S U(2)_{I} \times U(1)_{R}$. Their quantum numbers are in agreement with those of massive vectors in 6.11 .

\subsection{The $S U(2,2 \mid 1)$ spectrum and the operator map}

The ultimate test of our result for the mass spectrum is to verify that it can be assembled into irreducible unitary representations of the superalgebra $S U(2,2 \mid 1) \times S U(2)_{I}$. 
The relevant representations of $S U(2,2 \mid 1)$ are parametrized by the energy $E_{0}=\Delta$, of the lowest weight state, equal to the scaling dimension of the corresponding boundary operator, the two spins $j_{1}$ and $j_{2}$ and the $R$-charge, $r$. These quantum numbers designate a representation of the bosonic subalgebra $U(1) \times S U(2) \times S U(2) \times U(1)$ where the first and last $U(1)$ factors are the energy and $R$-symmetry, respectively. The relation between $\Delta$ and the mass for various fields may, for example, be found in [48] for $d=4$. In general, for $\mathrm{AdS}_{d+1}$, the relations are

(i) scalars [3]: $\quad \Delta_{ \pm}=\frac{1}{2}\left(d \pm \sqrt{d^{2}+4 m^{2}}\right)$,

(ii) spinors [49]: $\quad \Delta=\frac{1}{2}(d+2|m|)$,

(iii) vectors: $\Delta_{ \pm}=\frac{1}{2}\left(d \pm \sqrt{(d-2)^{2}+4 m^{2}}\right)$,

(iv) $p$-forms [50]: $\quad \Delta=\frac{1}{2}\left(d \pm \sqrt{(d-2 p)^{2}+4 m^{2}}\right)$,

(v) first-order $(d / 2)$-forms $\left(d\right.$ even): ${ }^{1} \quad \Delta=\frac{1}{2}(d+2|m|)$,

(vi) spin-3/2 [53,54]: $\quad \Delta=\frac{1}{2}(d+2|m|)$,

(vii) massless spin-2 [3]: $\quad \Delta=d$.

We have converted results for mass eigenvalues of all fluctuations into scale dimensions by the formulas. Results are tabulated in Tables 6.1 and 6.2 in which each horizontal band corresponds to a representation of the product of $S U(2,2 \mid 1)$ with the $S U(2)_{I}$ flavor symmetry. The detailed structure of these $S U(2,2 \mid 1)$ representations is given in Appendix B, and the entry in the tables denotes the lowest weight energy and the $S U(2)_{I} \times U(1)_{R}$ quantum numbers at each level. In all but one case we take $\Delta=\Delta_{+}$, the largest root given above. The unique exception is the complex scalar triplet with $\Delta=\Delta_{-}=3 / 2$. This assignment is required by the well known relation $\Delta=3 r / 2$ for $\mathcal{N}=1$ chiral primary operators and, of course, by the structure of the $S U(2,2 \mid 1)$ representation. The choice $\Delta=\Delta_{\text {- }}$ was made, also for group theoretic reasons, in another recent study [18] of the AdS/CFT correspondence. It is also known that the chiral multiplet in $A d S_{4}$ supersymmetry requires quantization with both "regular" and "irregular" boundary conditions [55] when scalar masses are in the range $-d^{2} / 4<m^{2}<1-d^{2} / 4$. See [56] for a discussion valid in $A d S_{d+1}$.

There is presently no coherent prescription to calculate correlation functions of scalar operators of dimension $\Delta<2$ from the AdS/CFT correspondence, and the two-point function appears to be particularly difficult. The following procedure seems a provisionally satisfactory application of the prescription of $[2,3]$. Consider the three-point correlator $\left\langle J_{\mu} \mathcal{O}^{*} \mathcal{O}\right\rangle$, where $\mathcal{O}$ is the operator of "irregular" dimension and $J_{\mu}$ is a conserved current of the boundary theory. In the present example $J_{\mu}$ could be one of the global $S U(2)$

\footnotetext{
${ }^{1}$ See [51] for $d=4$. Entry (v) is for forms with first order actions [52], while entries (iii) and (iv) are for forms with Maxwell type actions, possibly augmented by mass terms.
} 
currents or the R-symmetry current, both of which correspond to fields in five-dimensional gauged supergravity. Note that we do not specifically rely on supersymmetry here, but only on the existence of some conserved current under which $\mathcal{O}$ is charged. The three-point function above was studied in [57]. The required integral over $A d S_{5}$ converges for $\Delta>1$, exactly the range needed! The two-point function $\left\langle\mathcal{O}^{*} \mathcal{O}\right\rangle$ can be then obtained from the Ward identity for $J_{\mu}$, and it has the correct power law, namely $1 /|x-y|^{2 \Delta}{ }^{2}$

The short representations and corresponding superfields ${ }^{3}$ are listed in Table 6.1. They are either chiral, and thus satisfy $\Delta=3 r / 2$ for the lowest component, or they have protected dimensions because the multiplet contains one or more conserved component operators: for example, the $S U(2)$ flavor current in $\operatorname{Tr} \bar{\Phi} T^{A} \Phi$, and the $U(1)_{R}$ current, supercurrent, and stress tensor in the supercurrent superfield $J_{\alpha \dot{\alpha}}$. Since the massive field $\Phi_{3}$ is effectively integrated out in the flow, the IR theory contains the massless chiral superfields $\Phi_{i}, i=1,2$ with $\Delta=3 / 4$ and $r=1 / 2$ and the gauge superfields $W_{\alpha}$ with $\Delta=3 / 2$ and $r=1$. We see that the short multiplets found from $A d S_{5}$ supergravity contain all bilinears formed from these fields, all with correct quantum numbers. This is a striking confirmation of the validity of the correspondence in a new and more complex theory.

The first long multiplet in Table 6.2 is a massive vector representation of $S U(2,2 \mid 1)$ with irrational and clearly unprotected $\Delta$. We use the designation $K(x, \theta, \bar{\theta})$ to indicate that it corresponds to a general scalar superfield in the

\footnotetext{
${ }^{2}$ We thank E. Witten and L. Rastelli for useful discussion on this point.

${ }^{3}$ The relation between $S U(2,2 \mid 1)$ representations and boundary superfields in the context of the AdS/CFT correspondence has recently been discussed in [58, 59].
} 
boundary theory.

\begin{tabular}{lcllllll} 
& $\Delta$ & $\phi$ & $\chi$ & $A_{\mu}$ & $B_{\mu \nu}$ & $\psi_{\mu}$ & $h_{\mu \nu}$ \\
\hline $\mathcal{D}\left(\frac{3}{2}, 0,0 ; 1\right)$ & $\frac{3}{2}$ & $\mathbf{3}_{1}$ & & & & & \\
$\operatorname{Tr} \Phi_{i} \Phi_{j}$ & 2 & & $\mathbf{3}_{0}$ & & & & \\
$\operatorname{complex}$ & $\frac{5}{2}$ & $\mathbf{3}_{-1}$ & & & & \\
\hline $\mathcal{D}(2,0,0 ; 0)$ & 2 & $\mathbf{3}_{0}$ & & & & \\
$\operatorname{Tr} \bar{\Phi} T^{A} \Phi$ & $\frac{5}{2}$ & & $\mathbf{3}_{1} \oplus \mathbf{3}_{-1}$ & & & \\
$\operatorname{real}$ & 3 & & & $\mathbf{3}_{0}$ & & \\
\hline $\mathcal{D}\left(\frac{9}{4}, \frac{1}{2}, 0 ; \frac{3}{2}\right)$ & $\frac{9}{4}$ & & $\mathbf{2}_{3 / 2}$ & & & \\
$\operatorname{Tr} W_{\alpha} \Phi_{j}$ & $\frac{11}{4}$ & $\mathbf{2}_{1 / 2}$ & & & $\mathbf{2}_{1 / 2}$ & \\
$\operatorname{complex}$ & $\frac{13}{4}$ & & $\mathbf{2}_{-1 / 2}$ & & & \\
\hline $\mathcal{D}(3,0,0 ; 2)$ & 3 & $\mathbf{1}_{2}$ & & & & & \\
$\operatorname{Tr} W^{\alpha} W_{\alpha}$ & $\frac{7}{2}$ & & $\mathbf{1}_{1}$ & & & & \\
$\operatorname{complex}$ & 4 & $\mathbf{1}_{0}$ & & & & & \\
\hline $\mathcal{D}\left(3, \frac{1}{2}, \frac{1}{2} ; 0\right)$ & 3 & & & $\mathbf{1}_{0}$ & & & \\
$J_{\alpha \dot{\alpha}}$ & $\frac{7}{2}$ & & & & & $\mathbf{1}_{1} \oplus \mathbf{1}_{-1}$ & \\
$\operatorname{real}$ & 4 & & & & & $\mathbf{1}_{0}$ \\
\hline
\end{tabular}

Table 6.1: The five short $S U(2,2 \mid 1)$ representations in the mass spectrum of supergravity fields at the $\mathcal{N}=2$ critical point and the corresponding $\mathcal{N}=1$ superfields in the boundary gauge theory. For each complex representation above there is an additional representation with the same dimension, spin and $S U(2)_{I}$ content, but opposite $U(1)_{R}$ charge.

We would like to identify the scalar superfield $K(x, \theta, \bar{\theta})$ with an operator in the gauge theory. We speculate that it is the Kähler potential. This is a sensible guess because the Kähler potential evolves in the RG flow, and the scalar field that measures the approach of our trajectory to the $\mathcal{N}=1$ point sits in the supergravity multiplet dual to $K(x, \theta, \bar{\theta})$. This scalar field has a 
dimension $3+\sqrt{7}$ in the infrared.

\begin{tabular}{|c|c|c|c|c|c|c|}
\hline & $\Delta$ & $\phi$ & $\chi$ & $A_{\mu}$ & $B_{\mu \nu}$ & $\psi_{\mu}$ \\
\hline $\begin{array}{l}\mathcal{D}(\Delta, 0,0 ; 0) \\
\Delta=1+\sqrt{7} \\
K \\
\text { real }\end{array}$ & $\begin{array}{l}1+\sqrt{7} \\
\frac{3}{2}+\sqrt{7} \\
2+\sqrt{7} \\
\frac{5}{2}+\sqrt{7} \\
3+\sqrt{7}\end{array}$ & $\begin{array}{l}\mathbf{1}_{0} \\
\mathbf{1}_{2} \oplus \mathbf{1}_{-2} \\
\mathbf{1}_{0}\end{array}$ & $\begin{array}{l}\mathbf{1}_{1} \oplus \mathbf{1}_{-1} \\
\mathbf{1}_{1} \oplus \mathbf{1}_{-1}\end{array}$ & $\mathbf{1}_{0}$ & & \\
\hline $\begin{array}{l}\mathcal{D}\left(\frac{11}{4}, \frac{1}{2}, 0 ; \frac{1}{2}\right) \\
\Lambda_{\alpha i} \\
\text { complex }\end{array}$ & $\begin{array}{l}\frac{11}{4} \\
\frac{13}{4} \\
\frac{15}{4} \\
\frac{17}{4}\end{array}$ & $\mathbf{2}_{-1 / 2}$ & $\begin{array}{l}\mathbf{2}_{1 / 2} \\
\mathbf{2}_{1 / 2} \oplus \mathbf{2}_{-3 / 2}\end{array}$ & $\begin{array}{l}\mathbf{2}_{3 / 2} \\
\mathbf{2}_{-1 / 2}\end{array}$ & $\mathbf{2}_{-1 / 2}$ & $\mathbf{2}_{1 / 2}$ \\
\hline $\begin{array}{l}\mathcal{D}\left(3,0, \frac{1}{2} ; \frac{1}{2}\right) \\
\Sigma_{\alpha} \\
\text { complex }\end{array}$ & $\begin{array}{l}3 \\
\frac{7}{2} \\
4 \\
\frac{9}{2}\end{array}$ & & $\begin{array}{l}\mathbf{1}_{0} \\
\mathbf{1}_{-2}\end{array}$ & $\mathbf{1}_{-1}$ & $\begin{array}{l}\mathbf{1}_{1} \\
\mathbf{1}_{-1}\end{array}$ & $\mathbf{1}_{0}$ \\
\hline
\end{tabular}

Table 6.2: The remaining $S U(2,2 \mid 1)$ representations in the supergravity mass spectrum. The representations labelled complex are again doubled as described in the caption for Table 6.1.

The lowest weight states of the remaining long multiplets have spin $1 / 2$ and maximum spin $3 / 2$. We assign them to boundary operators which are non-chiral spinor superfields $\Lambda_{\alpha i}(x, \theta, \bar{\theta})$ and $\Sigma_{\alpha}(x, \theta, \bar{\theta})$. The natural candidates for these operators are the 3 supercurrents of the $\mathcal{N}=4$ theory which are explicitly broken by the mass term in (2.1). In the absence of the mass term the 3 extended supercurrents are components of the $\mathcal{N}=1 S U(3)$ triplet superfield ${ }^{4}$

$$
S_{\alpha}^{i}(x, \theta, \bar{\theta})=\operatorname{Tr}\left(W_{\alpha} \bar{\Phi}^{i}+\frac{1}{2} \epsilon^{i j k} \Phi_{j} D_{\alpha} \Phi_{k}\right),
$$

where $D_{\alpha}$ is the gauge and supercovariant derivative. We suggest that $\Lambda_{\alpha i}$ and $\Sigma_{\alpha}$ are the $i=1,2$ and $i=3$ components of this superfield, respectively, and we note that the $\mathrm{R}$-charges agree with this assignment. The scale dimensions $\Delta$ generally disagree with the naive sum of those of the elementary fields as is expected for non-chiral operators.

\footnotetext{
${ }^{4}$ We thank M. Grisaru for this information.
} 
The two massive spin-3/2 representations have strikingly different component structure, for example $\Lambda_{\alpha i}$ contains a scalar component, but $\Sigma_{\alpha}$ does not. We have confirmed in Appendix B that these representations are characterized by null or shortening conditions which eliminate states, specifically the condition $2 E_{0}=4\left(j_{2}+1\right)+3 r$ with $j_{2}=0$ for $\Sigma_{\alpha}$ and $2 E_{0}=4\left(j_{2}+1\right)+3 r$ with $j_{2}=\frac{1}{2}$ for $\Lambda_{\alpha i}$. Thus they have a protected structure in the same sense in which a chiral superfield with $2 E_{0}=3 r$ is protected. The structure of these representations suggests they should not be called long representations, but perhaps semi-long. The detailed correspondence between the supergravity fields which are components of these representations and the components of the superfields $S_{\alpha}^{i}$ is left here as a curious open question.

Although the detailed analysis of the representations of Table 6.2 is given in Appendix B, it is worth remarking here that their structures can be described in terms of the Higgs effect in which a massless multiplet of $S U(2,2 \mid 1)$, which contains a component gauge field, eats a lower spin multiplet. The massless representations are listed in Table 5 of [6]. The states of the massive vector multiplet we have called $K$ in Table 6.2 are exactly those of a massless vector multiplet plus a hypermultiplet ${ }^{5}$ in which one of the 4 real scalars is eaten. A massive spin- $3 / 2$ field has the choice of eating a spin- $1 / 2$ field from a vector or antisymmetric tensor multiplet. The states of the $\Sigma_{\alpha}$ representation are those of a massless gravitino multiplet plus a tensor multiplet with one spin- $1 / 2$ field and one scalar eaten to make its massive higher spin components. On the other hand the states of the $\Lambda_{\alpha i}$ representation are those of a gravitino plus vector plus LH spinor multiplet with a spinor and two scalars eaten. We have already seen that no Higgs mechanism is required to make an antisymmetric tensor massive.

\section{$7 \mathcal{N}=4$ supergravity}

Thus far, our explicit analysis of the $S U(2)_{I}$ invariant subsector of gauged $\mathcal{N}=8$ supergravity has focussed largely upon the scalar manifold. We now return to the consideration of the $\mathcal{N}=4$ supergravity. theory associated with the $S U(2)_{I}$ invariant subsector of the complete $\mathcal{N}=8$ theory.

The $\mathcal{N}=4$ supergravity in five dimensions was first considered in [60], but was only really explicitly described in [61]. The latter reference also considers the coupling to an arbitrary number, $m$, of vector multiplets, and goes on to discuss the gauged theory with an $S U(2)$ gauge group. Various

\footnotetext{
${ }^{5} \mathrm{~A}$ hypermultiplet is the direct sum of a $\mathrm{LH}$ and $\mathrm{RH}$ spinor multiplet in the notation of $[6]$.
} 
gaugings of pure $\mathcal{N}=4$ supergravity (i.e. not coupled to vector or tensor multiplets) were also considered in [62].

The field content of the $\mathcal{N}=4$ graviton multiplet is one gravitino, four gravitinos, six vectors, four spin- $\frac{1}{2}$ fields and a single, real scalar. The content of the $\mathcal{N}=4$ vector multiplet is: one vector, four spin- $\frac{1}{2}$ fields and five real scalars. The scalar manifold of the supergravity coupled to $m$ vector multiplets is:

$$
\frac{S O(5, m)}{S O(5) \times S O(m)} \times S O(1,1)
$$

where the factor of $S O(1,1)$ represents the scalar of the graviton multiplet.

If one truncates the ungauged $\mathcal{N}=8$ theory to the singlets of $S U(2)_{I}$ one gets precisely $\mathcal{N}=4$ supergravity coupled to two $\mathcal{N}=4$ vector multiplets. However the truncation of the $S O(6)$ gauged supergravity yields something a little different from that of [61] because the residual gauge group is $S U(2)_{G} \times$ $U(1)_{G}$ and not just $S U(2)$. The gauging of the extra $U(1)$ appears to require the dualization of some of the vector fields into tensor gauge fields just as in [6]. One can easily determine the field content and compute the structure of the field theory by truncation the results of $[6]$ to the $S U(2)_{I}$ singlet sector. Indeed, the four vectors 3.3 can be used to project the $U S p(8)$ structure appropriately. To be explicit:

(i) The gravitinos of the $\mathcal{N}=8$ theory transform $4 \oplus \overline{4}$ of $S U(4)$. Under $S U(2)_{I} \times S U(2)_{G} \times U(1)_{G}$, one has $\mathbf{4}=(\mathbf{2}, \mathbf{1})\left(+\frac{1}{2}\right) \oplus(\mathbf{1}, \mathbf{2})\left(-\frac{1}{2}\right)$. Thus there are four gravitinos that are singlets under this $S U(2)_{I}$, transforming as $\mathbf{2}\left(-\frac{1}{2}\right) \oplus \mathbf{2}\left(+\frac{1}{2}\right)$ of $S U(2)_{G} \times U(1)_{G}$. They are explicitly given by the inner products of 3.3 and the $\mathcal{N}=8$ gravitinos. The supersymmetries decompose similarly.

(ii) The vector fields are in the $\mathbf{1 5}$ of $S U(4)$ and the $S U(2)_{I}$ singlets are simply the adjoints of $S U(2)_{G} \times U(1)_{G}$.

(iii) The tensor gauge fields are in an $S L(2, \mathbb{R})$ doublet of 6 's of $S U(4)$ : and each member of the $S L(2, \mathbb{R})$ doublet gives rise to two tensor gauge fields that are singlets of $S U(2)_{G}$ but have charges of \pm 1 under $U(1)_{G}$.

(iv) The spin- $\frac{1}{2}$ fields are in the following representations of $S U(2)_{G} \times$ $U(1)_{G}: \mathbf{2}\left(-\frac{3}{2}\right) \oplus \mathbf{2}\left(+\frac{3}{2}\right) \oplus 2 \times \mathbf{2}\left(-\frac{1}{2}\right) \oplus 2 \times \mathbf{2}\left(+\frac{1}{2}\right)$

(v) The eleven scalars were described above.

Putting this together, the theory consists of an $\mathcal{N}=4$ graviton supermultiplet and two $\mathcal{N}=4$ tensor gauge super multiplets. The former has: one 
graviton, four gravitinos, four vector fields, two tensor gauge fields, four spin- $\frac{1}{2}$ fields, and one real scalar. The tensor gauge multiplet has the same spin- $\frac{1}{2}$ and scalar content as an $\mathcal{N}=4$ vector multiplet.

The truncation process can be viewed in two ways. Mathematically one is identifying a subset of fields whose Lagrangian and equations of motion involve only those fields, and such that any solution of the truncated equations of motion can be lifted to a solution of the untruncated equations of motion. Perhaps the simplest way of finding such a truncation is by restricting to the fields that are singlets under some particular symmetry (here it is $S U(2)_{I}$ ), and then Schur's lemma guarantees consistency with the equations of motion of the untruncated theory. Physically the truncated theory can be taken as a new field theory in its own right, and it is consistent and well-defined at least at the classical level. Or one can regard it as part of the original (or perhaps another) larger field theory.

Naively, one might hope that the $\mathcal{N}=4$ truncation of gauged $\mathcal{N}=8$ supergravity corresponds to a truncation of $\mathcal{N}=4$ Yang-Mills to its $S U(2)_{I}$ singlet sector. However, the $S U(2)_{I}$ invariant subsector of $\mathcal{N}=4$ Yang-Mills is simply a pure $\mathcal{N}=2$ Yang-Mills theory, which is not a superconformal field theory, and so cannot, by itself be holographically dual to the $\mathcal{N}=4$ supergravity theory. The error in this naive hope is that fundamental fields of supergravity are not dual to fundamental fields of Yang-Mills, and so one must proceed more carefully. In other words, the truncation to the $S U(2)_{I}$ invariant sector must be applied to the gauge invariant composite operators rather than to the fundamental colored fields themselves.

There are however many ways to build a superconformal field theory around the same set of $S U(2)_{I}$ invariant composite operators dual to the truncated $\mathcal{N}=4$ supergravity theory. The focus of this paper has been on adding adjoint hypermultiplets to make the $\mathcal{N}=4$ theory; however there are also the $\mathcal{N}=2$ superconformal gauge theories whose gauge groups and matter multiplets are encoded in an ADE quiver diagram [25]. Using the $D$-brane formulation of $\mathcal{N}=4$ Yang-Mills theory one can make an orbifold construction $[63,26]$ using a finite subgroup $\Gamma$ of $S U(2)_{I}$. These finite subgroups also have an ADE classification, and indeed the ADE orbifold leads to the corresponding $\mathrm{ADE} \mathcal{N}=2$ "quiver theory." The common subsector of all these conformal field theories is the $\Gamma$ invariant gauge-singlet operators, corresponding holographically to untwisted closed string states. This is in a sense the $\mathcal{N}=2$ Yang-Mills "core" of the theory. It is thus tempting to identify this universal subsector of the conformal field theories as the holographic dual of $\mathcal{N}=4$ supergravity coupled to two tensor multiplets. Inclusion of the twisted sectors may involve further coupling to additional 
$\mathcal{N}=4$ supermultiplets. The truncated $\mathcal{N}=4$ supergravity is dual to the stress tensor of the full superconformal field theory plus its superpartners under $\mathcal{N}=2$ superconformal invariance.

To see more explicitly how this should work, observe that the 11 scalars of $\mathcal{N}=4$ supergravity coupled to two tensor multiplets provides precisely the proper moduli of the $\mathcal{N}=2$ Yang-Mills vector multiplet: that is, the gauge coupling, the $\theta$-angle, a real, symmetric $2 \times 2$ mass matrix for the two real scalars and a complex, symmetric $2 \times 2$ mass matrix for the two complex fermions. The $S U(2) \times U(1) R$-symmetry can then be used to reduce the nine mass parameters to five, and these can be represented by diagonal mass matrices with real traces. The $U(1)$ axial symmetry can be used to reduce this by one further parameter, leaving four independent mass perturbations, which correspond to the four independent scalars described in [8].

If $\mathcal{N}=4$ supergravity coupled to two $\mathcal{N}=4$ tensor multiplets does indeed provide the common core of all the quiver models, then the scalar manifold $\mathcal{S}$ considered in [8] is not only part of the phase diagram of $\mathcal{N}=4$ Yang-Mills, but is that part of the phase diagram that is common to $\mathcal{N}=4$ Yang-Mills and to all the $\mathcal{N}=2$ quiver theories. The critical points and flows described here would thus also be common to all these theories. 


\section{APPENDIX A. $S U(2)$ invariant scalar manifolds in $\mathcal{N}=8$ supergravity}

In this appendix we review the construction of the $S U(2)$ invariant sectors of the scalar manifold of $\mathcal{N}=8 d=5$ gauged supergravity which played a central role in [8]. In particular, we derive an explicit parametrization of the manifold $\mathcal{S}$ in section 3 and collect some explicit formulae which are used in the computation of the mass spectra in section 6 .

The scalar fields in $\mathcal{N}=8$ supergravity are described by a non-linear $\sigma$-model on the non-compact coset space $E_{6(6)} / U S p(8)[60]$. In the gauged theory $[5,6,7]$ the global symmetry group $E_{6(6)}$ is broken to an $S O(6)$ subgroup, which then becomes the local gauge symmetry group of the theory. The authors of [8] considered those scalar field configurations which are invariant under an $S U(2)$ subgroup of $S O(6)$ that we call $S U(2)_{I}$. Let $C$ be the commutant of $S U(2)_{I}$ in $E_{6(6)}$ and $K \subset C$ the maximal compact subgroup of $C$. Then the reduced scalar manifold $\mathcal{S}$ corresponding to a given $S U(2)_{I}$ is

$$
\mathcal{S} \simeq C / K \subset E_{6(6)} / U S p(8) .
$$

The commutant of $S U(2)_{I}$ in $S O(6)$ is the group of residual gauge transformations on $\mathcal{S}$, which will be denoted by $G$.

The scalar potential, $V$, restricted to the fields in $\mathcal{S}$, is invariant under the residual gauge symmetry, $G$, and, in addition, under $S L(2, \mathbb{R})$ of the tendimensional theory. Because of this invariance the potential restricted to $\mathcal{S}$ actually depends on a reduced number of independent parameters, denoted by $\operatorname{dim} V$ which is less than $\operatorname{dim} S$.

There are four inequivalent embeddings of $S U(2)_{I} \hookrightarrow S O(6)$ corresponding to the following branchings of the fundamental and the vector representations of $S O(6)$ :

\begin{tabular}{llllll}
$S U(2)_{I}$ & $C$ & $K$ & $G$ & $\operatorname{dim} \mathcal{S}$ & $\operatorname{dim} V$ \\
\hline (i) & $S O(5,2) \times O(1,1)$ & $S O(5) \times S O(2)$ & $S U(2) \times U(1)$ & 11 & 4 \\
(ii) & $S L(3, \mathbb{R}) \times S L(3, \mathbb{R})$ & $S O(3) \times S O(3)$ & $S U(2)$ & 10 & 4 \\
(iii) & $G_{2(2)}$ & $S O(3) \times S O(3)$ & $U(1) \times U(1)$ & 8 & 4 \\
(iv) & $O(1,1) \times S L(2, \mathbb{R})$ & - & - & 4 & 1
\end{tabular}

Table A.1: Scalar manifolds, $\mathcal{S}$, for inequivalent embeddings $S U(2)_{I} \hookrightarrow S O(6)$.

Our starting point is a real 27-dimensional realizations of $E_{6(6)}$ in the $S L(6, \mathbb{R}) \times S L(2, \mathbb{R})$ basis $^{1}\left(z_{I J}, z^{I \alpha}\right), z_{I J}=-z_{J I}$, as described in $[60]$ and,

\footnotetext{
${ }^{1}$ We use the notation $I, J, \ldots=1, \ldots, 6$ and $\alpha, \beta, \ldots=1,2$ for the $S L(6, \mathbb{R})$ and $S L(2, \mathbb{R})$ indices, respectively.
} 
in particular, in Appendix A of [6], which the reader should consult for conventions and further details. An infinitesimal $E_{6(6)}$ action on $\left(z_{I J}, z^{I \alpha}\right)$ is given by

$$
\begin{aligned}
& \delta z_{I J}=-\Lambda^{K}{ }_{I} z_{K J}-\Lambda^{K}{ }_{J} z_{I K}+\Sigma_{I J K \beta} z^{K \beta} \\
& \delta z^{I \alpha}=\Lambda^{I}{ }_{K} z^{K \alpha}+\Lambda^{\alpha}{ }_{\beta} z^{I \beta}+\Sigma^{K L I \alpha} z_{K L}
\end{aligned}
$$

where $\Lambda_{J}^{I}, \Lambda_{\beta}^{\alpha}$ and $\Sigma_{I J K \alpha}=\frac{1}{6} \epsilon_{I J K L M N} \epsilon_{\alpha \beta} \Sigma^{L M N \beta}$ correspond to $S L(6, \mathbb{R})$, $S L(2, \mathbb{R})$ and the coset elements, respectively. The antisymmetric matrices $\Lambda^{I}{ }_{J}=-\Lambda_{I}^{J}$ generate the gauge group $S O(6)$.

Define $4 \times 4$ 't Hooft matrices

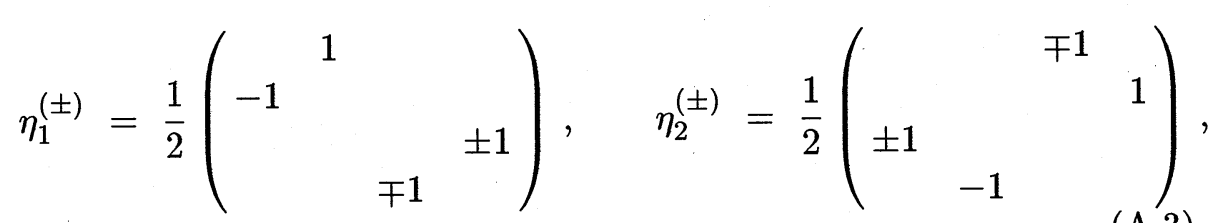

$$
\begin{aligned}
& \eta_{3}^{( \pm)}=\frac{1}{2}\left(\begin{array}{lll} 
& & \\
-1 & &
\end{array}\right)
\end{aligned}
$$

satisfying

$$
\left[\eta_{r}^{(+)}, \eta_{s}^{(-)}\right]=0, \quad\left[\eta_{r}^{( \pm)}, \eta_{s}^{( \pm)}\right]=\epsilon_{r s t} \eta_{t}^{( \pm)}
$$

and

$$
\frac{1}{2} \epsilon_{i j k l} \eta_{r}^{( \pm) k l}= \pm \eta_{r}^{( \pm) i j}
$$

The $S U(2)_{I}$ in case (i) is generated by the $S O(6)$ matrices

$$
\left(\Lambda_{J}^{I}\right)=\left(\begin{array}{ccc}
\eta_{r}^{(-)} & 0 & 0 \\
0 & 0 & 0 \\
0 & 0 & 0
\end{array}\right), \quad r=1,2,3
$$

The residual gauge group is $S U(2)_{G} \times U(1)_{G}$, where $S U(2)_{G}$ is generated by the $\eta_{r}^{(+)}$in the same $4 \times 4$ block as above, so that $S U(2)_{I} \times S U(2)_{G}$ is the obvious $S O(4)$ subgroup of $S O(6)$, while $U(1)_{G}$ are the $S O(2)$ rotations in the 56-block.

Now, let us introduce a new basis in which the $S O(5,2)$ transformations acquire a canonical form. A helpful observation here is the branching of the 27 of $E_{6(6)}$ under $S U(2)_{I} \times S O(5,2) \times O(1,1)$,

$$
\mathbf{2 7} \rightarrow(\mathbf{1}, \mathbf{1})(-4) \oplus(\mathbf{3}, \mathbf{1})(2) \oplus(\mathbf{1}, \mathbf{7})(2) \oplus(\mathbf{2}, \mathbf{8})(-1) .
$$


The corresponding new basis is explicitly given by

$$
\begin{aligned}
& u_{(1,1)}=z_{56} ; \\
& u_{(3,1)}^{1}=\frac{1}{\sqrt{2}}\left(z_{12}-z_{34}\right), u_{(3,1)}^{2}=\frac{1}{\sqrt{2}}\left(z_{13}+z_{24}\right), u_{(3,1)}^{3}=\frac{1}{\sqrt{2}}\left(z_{14}-z_{23}\right) ;(\mathrm{A} .9) \\
& u_{(1,7)}^{1}=-\frac{1}{\sqrt{2}}\left(z_{12}+z_{34}\right), u_{(1,7)}^{2}=\frac{1}{\sqrt{2}}\left(z_{13}-z_{24}\right), u_{(1,7)}^{3}=-\frac{1}{\sqrt{2}}\left(z_{14}+z_{23}\right), \\
& u_{(1,7)}^{4}=\frac{1}{2}\left(z^{51}-z^{62}\right), u_{(1,7)}^{5}=\frac{1}{2}\left(z^{52}+z^{61}\right), u_{(1,7)}^{6}=\frac{1}{2}\left(z^{51}+z^{62}\right), \\
& u_{(1,7)}^{7}=-\frac{1}{2}\left(z^{52}-z^{61}\right) ; \\
& u_{(2,8)}^{11}=\frac{1}{\sqrt{2}}\left(z_{15}-i z_{25}\right), u_{(2,8)}^{12}=\frac{1}{\sqrt{2}}\left(z_{16}-i z_{26}\right), u_{(2,8)}^{13}=\frac{1}{\sqrt{2}}\left(z_{35}+i z_{45}\right), \\
& u_{(2,8)}^{14}=\frac{1}{\sqrt{2}}\left(z_{36}+i z_{46}\right), u_{(2,8)}^{15}=\frac{1}{2}\left(z^{21}+i z^{11}\right), u_{(2,8)}^{16}=\frac{1}{2}\left(z^{22}+i z^{12}\right),(\mathrm{A} .11) \\
& u_{(2,8)}^{17}=\frac{1}{2}\left(z^{41}-i z^{31}\right), u_{(2,8)}^{18}=\frac{1}{2}\left(z^{42}-i z^{32}\right) ; \\
& u_{(2,8)}^{21}=-\frac{1}{\sqrt{2}}\left(z_{35}-i z_{45}\right), u_{(2,8)}^{22}=-\frac{1}{\sqrt{2}}\left(z_{36}-i z_{46}\right), u_{(2,8)}^{23}=\frac{1}{\sqrt{2}}\left(z_{15}+i z_{25}\right), \\
& u_{(2,8)}^{24}=\frac{1}{\sqrt{2}}\left(z_{16}+i z_{26}\right), u_{(2,8)}^{25}=-\frac{1}{2}\left(z^{41}+i z^{31}\right), \\
& u_{(2,8)}^{26}=-\frac{1}{2}\left(z^{42}+i z^{32}\right) u_{(2,8)}^{27}=\frac{1}{2}\left(z^{21}-i z^{11}\right), u_{(2,8)}^{28}=\frac{1}{2}\left(z^{22}-i z^{12}\right) .
\end{aligned}
$$

We will denote the elements of this basis by $u^{\Omega}, \Omega=1, \ldots, 27$ and define the transition matrix $O$,

$$
z_{I J}=O_{I J \Omega} u^{\Omega}, \quad z^{I \alpha}=O_{\Omega}^{I \alpha} u^{\Omega},
$$

and its inverse $\widetilde{O}$,

$$
u^{\Omega}=\frac{1}{2} \widetilde{O}^{\Omega I J} z_{I J}+\widetilde{O}_{I \alpha}^{\Omega} z^{I \alpha} .
$$

The eleven-dimensional family of infinitesimal noncompact transformations of the group $S O(5,2) \times O(1,1)$ is parametrized by $x^{i}, y^{i}, i=1, \ldots, 5$ and $\alpha$, such that

$$
\begin{gathered}
\left(\Lambda_{J}^{I}\right)=\operatorname{diag}\left(-\alpha,-\alpha,-\alpha,-\alpha,\left(\begin{array}{cc}
2 \alpha-x^{4}+y^{5} & -x^{5}-y^{4} \\
-x^{5}-y^{4} & 2 \alpha+x^{4}-y^{5}
\end{array}\right)\right) \\
\left(\Lambda_{\beta}^{\alpha}\right)=\left(\begin{array}{cc}
-x^{4}-y^{5} & -x^{5}+y^{4} \\
-x^{5}+y^{4} & x^{4}+y^{5}
\end{array}\right)
\end{gathered}
$$

and

$$
\Sigma^{I J 51}=\Sigma^{I J 62}=\sum_{i=1}^{3} \sqrt{2} x^{i} \eta_{i}^{I J}
$$




$$
\Sigma^{I J 61}=-\Sigma^{I J 52}=\sum_{i=1}^{3} \sqrt{2} y^{i} \eta_{i}^{I J}, \quad I, J=1, \ldots, 4,
$$

where $\eta_{r}=\eta_{r}^{(+)}$. It is now easy to verify that these transformations commute with $S U(2)_{I}$. Moreover, we find that in the new basis they become

$$
\begin{gathered}
\delta u_{(1,1)}=-4 \alpha u_{(1,1)}, \\
\delta u_{(3,1)}^{\rho}=2 \alpha u_{(3,1)}^{\rho}, \\
\delta u_{(1,7)}^{i}=2 \alpha u_{(1,7)}^{i}+M_{7}(x, y)^{i}{ }_{j} u_{(1,7)}^{j},
\end{gathered}
$$

and

$$
\delta u_{(2,8)}^{1,2}=-\alpha u_{(2,8)}^{1,2}+M_{8}(x, y) u_{(2,8)}^{1,2}
$$

where

$$
M_{8}(x, y)=\sum_{i=1}^{5}\left(x^{i} \tilde{\Gamma}_{i} \tilde{\Gamma}_{6}+y^{i} \tilde{\Gamma}_{i} \tilde{\Gamma}_{7}\right)
$$

and $M_{7}(x, y)$ is the corresponding vector matrix

$$
M_{7}(x, y)=-2\left(\begin{array}{ccc}
0 & x & y \\
x^{T} & 0 & 0 \\
y^{T} & 0 & 0
\end{array}\right)
$$

satisfying

$$
M_{8}(x, y) \tilde{\Gamma}_{i}-\tilde{\Gamma}_{i} M_{8}(x, y)=M_{7}(x, y)^{j}{ }_{i} \tilde{\Gamma}_{j}
$$

The $S O(5,2)$ gamma matrices $\tilde{\Gamma}_{i}, i=1, \ldots, 7$, are given in Appendix C. Note that $u_{(2,8)}^{1}$ and $u_{(2,8)}^{2}$ form a pair of symplectic Majorana spinors, $u_{(2,8)}^{2}=$ $i \tilde{\Gamma}_{2}\left(u_{(2,8)}^{1}\right)^{*}$, as required by the reality of the 27 of $E_{6(6)}$.

Let us write A.18-A.21 in the matrix form $\delta u^{\Omega}=M(x, y, \alpha)^{\Omega} U^{\Sigma}$. The reduced scalar manifold $\mathcal{S}$ consists of finite transformations

$$
D(x, y, \alpha)=\exp M(x, y, \alpha),
$$

and by the standard properties of the non-compact cosets [64] has global coordinates $x^{i}, y^{i}$ and $\alpha$.

To define the 27-bein on $\mathcal{S}$ we introduce yet another basis, $z^{a b}, a ; b=$ $1, \ldots, 8$, transforming in the $\mathbf{2 7}$ of $U S p(8)$. It is defined in terms of the $S L(6, \mathbb{R}) \times S L(2, \mathbb{R})$ basis by (see $(\mathrm{A} .45)$ of $[6]$ )

$$
z^{a b}=\frac{1}{4}\left(\Gamma_{I J}\right)^{a b} z_{I J}+\frac{1}{2 \sqrt{2}}\left(\Gamma_{I \alpha}\right)^{a b} z^{I \alpha},
$$


where the gamma matrices $\Gamma_{I J}$ and $\Gamma_{I \alpha}$ are given in Appendix C.1. By combining transformations between the various bases, we find that the 27bein $\left(\mathcal{V}^{I J a b}, \mathcal{V}_{I \alpha}{ }^{a b}\right)$ on $\mathcal{S}$ is

$$
\mathcal{V}^{I J a b}=\frac{1}{2} G^{a b}{ }_{\Omega} D^{\Omega}{ }_{\Sigma} \widetilde{O}^{\Sigma I J}, \quad \mathcal{V}_{I \alpha}^{a b}=G^{a b}{ }_{\Omega} D^{\Omega}{ }_{\Sigma} \widetilde{O}^{\Sigma}{ }_{I \alpha},
$$

where

$$
G_{\Omega}^{a b}=\frac{1}{4}\left(\Gamma_{I J}\right)^{a b} O_{I J \Omega}+\frac{1}{2 \sqrt{2}}\left(\Gamma_{I \alpha}\right)^{a b} O_{\Omega}^{I \alpha},
$$

while the inverse 27-bein is

$$
\tilde{\mathcal{V}}_{a b I J}=O_{I J \Omega} \widetilde{D}_{\Sigma}^{\Omega} \widetilde{G}_{a b}^{\Sigma}, \quad \widetilde{\mathcal{V}}_{a b}^{I \alpha}=O^{I \alpha}{ }_{\Omega} \widetilde{D}_{\Sigma}^{\Omega} \widetilde{G}_{a b}^{\Sigma},
$$

with

$$
\widetilde{G}_{a b}^{\Omega}=\frac{1}{8} \widetilde{O}^{\Omega I J}\left(\Gamma_{I J}\right)^{a b}-\frac{1}{2 \sqrt{2}} \widetilde{O}_{I \alpha}^{\Omega}\left(\Gamma_{I \alpha}\right)^{a b} .
$$

The potential, $V$, of gauged $\mathcal{N}=8$ is defined in terms of $U S p(8)$ tensors

$$
W_{a b c d}=\epsilon^{\alpha \beta} \delta^{I J} \mathcal{V}_{\text {I } a b b} \mathcal{V}_{J \beta c d}, \quad W_{a b}=W_{a c b}^{c},
$$

where the $U S p(8)$ indices are lowered using the symplectic metric $\Omega_{a b}$. We have [6]

$$
V=-\frac{1}{32} g^{2} \cdot\left(2\left(W_{a b}\right)^{2}-\left(W_{a b c d}\right)^{2}\right) .
$$

In general, the potential, $V$, is invariant under the $G_{R} \times G_{L}$ group of local transformations of the 27-bein, where $G_{R}=U S p(8)$ and $G_{L}=S O(6) \times$ $S L(2, \mathbb{R})$. Upon restriction to the scalar submanifold, $\mathcal{S}$, a subgroup of those transformations

$$
D \quad \rightarrow \quad G^{\prime} D G^{\prime-1} P,
$$

preserves $\mathcal{S}$ and leaves $V$ invariant. The transformations in $G^{\prime}$ act linearly on $x^{i}$ and $y^{i}$. In terms of the $5 \times 2$ matrix $(x, y)$ in A.23 this action is schematically of the form

$$
(x, y) \rightarrow\left(\begin{array}{ll}
R_{S O(3)} & \\
& R_{S O(2)}
\end{array}\right)(x, y)\left(R_{S O(2)^{\prime}}\right),
$$

where the $S O(3)$ subgroup are the $S O(5,2)$ rotations in the (123)-hyperplane, the $S O(2)$ are the rotations in the (45)-plane and $S O(2)^{\prime}$, which rotates $\left(x^{i}, y^{i}\right)$ as a doublet, are the rotations in the (67)-plane. The group of residual gauge transformations, $G$, in Table A.1 consists of $S O(3)$ and a diagonal $\mathrm{SO}(2)$ in $S O(2) \times S O(2)^{\prime}$ above. Another combination of the $S O(2)$ rotations is a part of $S L(2, \mathbb{R})$. An infinitesimal action of $P$, the noncomapct generators of $S L(2, \mathbb{R})$, with parameters $\varepsilon^{1}$ and $\varepsilon^{2}$ on $(x, y)$ is given by

$$
\left(\begin{array}{ll}
x^{4} & y^{4} \\
x^{5} & y^{5}
\end{array}\right) \rightarrow\left(\begin{array}{cc}
x^{4}+\varepsilon^{1} & y^{4}+\varepsilon^{2} \\
x^{5}-\varepsilon^{2} & y^{5}+\varepsilon^{1}
\end{array}\right) .
$$


It was argued in [8] that using those invariances one may set all parameters, but $x^{1}, x^{4}, y^{2}$ and $\alpha$, to zero, thus reducing the dependence of the potential from 11 to 4 parameters. This simplification allowed the authors of [8] to compute the potential explicitly in terms of the variables $r_{x}, r_{y}, \theta$ and $\alpha$, where

$$
x^{1}=r_{x} \cos \theta, \quad x^{4}=r_{x} \sin \theta, \quad y^{2}=r_{y} .
$$

The $\mathcal{N}=2$ critical point is at $r_{x}=r_{y}= \pm \frac{1}{4} \log (3), \theta=0$ and $\alpha=\alpha_{0}=$ $\frac{1}{6} \log (2)$. It is invariant under $S U(2)_{I} \times U(1)_{R}$ subgroup of $S O(6)$, where the $R$-charge subgroup $U(1)_{R}$ is an unbroken subgroup of $S U(2)_{G} \times U(1)_{G}$. To identify this subgroup we note that the matrix $M(x, y, \alpha)$ at the critical point, when expressed in the $S L(6, \mathbb{R}) \times S L(2, \mathbb{R})$ basis using A.26-A.28, is given by

$$
\left(\Lambda_{J}^{I}\right)=\operatorname{diag}\left(-\alpha_{0},-\alpha_{0},-\alpha_{0},-\alpha_{0}, 2 \alpha_{0}, 2 \alpha_{0}\right), \quad\left(\Lambda_{\beta}^{\alpha}\right)=(0),
$$

and

$$
\begin{aligned}
& \Sigma^{I J 51}=\Sigma^{I J 62}= \pm \frac{1}{2 \sqrt{2}} \log (3) \eta_{1}^{(+) I J} \\
& \Sigma^{I J 61}=-\Sigma^{I J 52}= \pm \frac{1}{2 \sqrt{2}} \log (3) \eta_{2}^{(+) I J}
\end{aligned}
$$

The only nontrivial condition is that the $\Sigma$ 's above are invariant under $U(1)_{R}$, which determines the $R$-charge generator

$$
Y_{R}=\left(\Lambda_{J}^{I}\right)=\left(\begin{array}{ccc}
\eta_{3}^{(+)} & 0 & 0 \\
0 & 0 & -1 \\
0 & 1 & 0
\end{array}\right)
$$

It has been normalized such that under $S U(2)_{I} \times U(1)_{R} \hookrightarrow S O(6)$ we have

$$
6 \quad \rightarrow \quad \mathbf{2}_{1 / 2} \oplus \mathbf{2}_{-1 / 2} \oplus \mathbf{1}_{1} \oplus \mathbf{1}_{-1} \text {. }
$$

Finally, we compute the $U S p(8)$ tensors $W_{a b}, W_{a b c d}$ and

$$
A_{a b c d}=-3 W_{a[b c d] \mid},
$$

at the critical point. In particular,

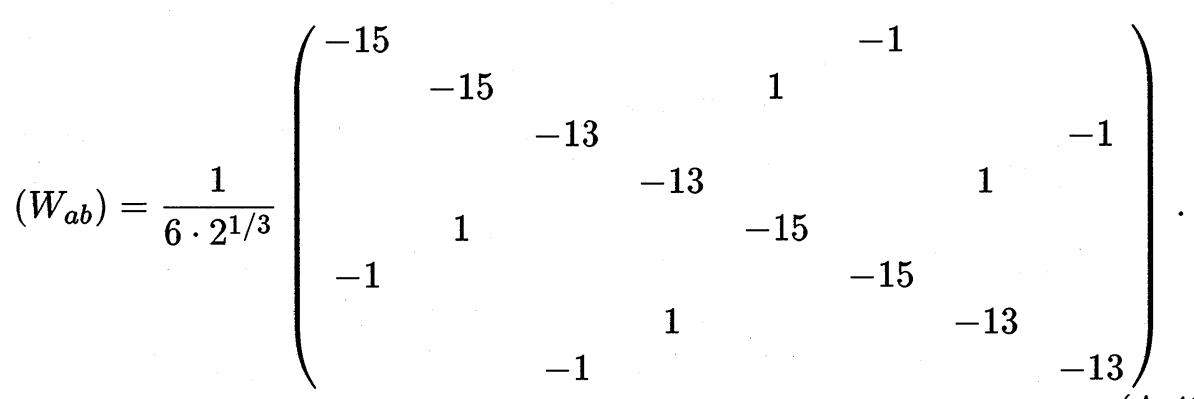




\section{APPENDIX B. Unitary multiplets of $S U(2,2 \mid 1)$}

In this appendix we recall the classification of the unitary highest weight representations of the superalgebra $S U(2,2 \mid 1)$ in $[45,46]$ (see, also [65]) and work out explicitly shortening patterns for the multiplets that are relevant for the discussion in section 6 .

A unitary highest weight representation of $S U(2,2 \mid 1)$ can be decomposed into a direct sum of unitary highest weight representations of its bosonic subalgebra $S U(2,2) \times U(1)$. The latter has a maximal compact subalgebra $U(1) \times S U(2) \times S U(2) \times U(1)$, in which the generator of the first $U(1)$ factor is the energy and that of the second is $R$-charge. The two $S U(2)$ factors give the obvious $S O(4)$ subalgebra of $S U(2,2) \simeq S O(4,2)$. Highest weight representations, $\mathcal{D}\left(E_{0}, j_{1}, j_{2} ; r\right)$, of $S U(2,2 \mid 1)$ are parametrized by the corresponding four quantum numbers.

Theorem $[45,46]$. A representation, $\mathcal{D}\left(E_{0}, j_{1}, j_{2} ; r\right)$, is unitary if and only if one of the following conditions (i)-(iv) holds:

(i) $E_{0} \geq 2 j_{1}-\frac{3}{2} r+2$ and $E_{0} \geq 2 j_{2}+\frac{3}{2} r+2$ for $j_{1}, j_{2} \geq 0$,

(ii) $E_{0}=-\frac{3}{2} r$ and $E_{0} \geq 2 j_{2}+\frac{3}{2} r+2$ for $j_{1}=0$ and $j_{2} \geq 0$,

(iii) $E_{0}=\frac{3}{2} r$ and $E_{0} \geq 2 j_{1}-\frac{3}{2} r+2$ for $j_{1} \geq 0$ and $j_{2}=0$,

(iv) $E_{0}=r=0$ for $j_{1}=j_{2}=0$.

Maximal representations (long multiplets) consist of the 16 representations of $S U(2,2)$ listed in the second column of Table B.1, each with multiplicity 1 . One may notice that each representation in the multiplet is uniquely identified by its $\left(j_{1}, j_{2} ; r\right)$. The level in the first column of the table is the minimal number of supersymmetry operators that must be applied to the vacuum to reach a given representation.

Shorter multiplets arise when either $j_{1} j_{2}=0$, or, more interestingly, when some of the equalities in the unitarity bounds (i)-(iii) are saturated. In the latter case some of the descendant $S U(2,2)$ representations that are predicted by the tensor product rules are missing. A complete list of shortening patterns is given in [45]. One can succinctly summarize the structure of the resulting short modules as follows:

Corollary.Define $N(x)=1$ for $x \neq 0$ and $N(0)=0$. Let

$$
\begin{aligned}
& n_{1}^{(+)}=N\left(E_{0}+2 j_{1}+\frac{3}{2} r\right), \quad n_{1}^{(-)}=N\left(E_{0}-2 j_{1}+\frac{3}{2} r-2\right),( \\
& n_{2}^{(+)}=N\left(E_{0}+2 j_{2}-\frac{3}{2} r\right), \quad n_{2}^{(-)}=N\left(E_{0}-2 j_{2}-\frac{3}{2} r-2\right),
\end{aligned}
$$


and

$$
s_{1}=N\left(j_{1}\right), \quad s_{2}=N\left(j_{2}\right) .
$$

The multiplicities of the $S U(2,2)$ representations in the decomposition of a unitary representation, $\mathcal{D}\left(E_{0}, j_{1}, j_{2} ; r\right)$, are determined by B.1, B.2 and the appropriate products of these numbers as given in Table B.1.

The short multiplets in Table 6.1 in section 6 are either chiral (complex) or non-chiral (real). The shortening conditions for the chiral multiplets are:

(R) $E_{0}=-\frac{3}{2} r$ and $j_{1}=0$ for the RH-multiplets,

(L) $E_{0}=\frac{3}{2} r$ and $j_{2}=0$ for the LH-multiplets.

We then have $n_{1}^{(+)}=0$ and $n_{2}^{(+)}=0$, respectively. For a generic value of $E_{0}$, a LH-multiplet is a direct sum of four $S U(2,2)$ multiplets for $j>0$ and three multiplets for $j=0$ as shown in Table B.2. The RH-multiplets are obtained by $\left(j_{1}, j_{2}\right) \rightarrow\left(j_{2}, j_{1}\right)$ and $r \rightarrow-r$.

\begin{tabular}{lll} 
Level & SU(2,2)representation & Multiplicity \\
\hline 0 & $D\left(E_{0}, j_{1}, j_{2} ; r\right)$ & 1 \\
\hline 1 & $D\left(E_{0}+\frac{1}{2}, j_{1}+\frac{1}{2}, j_{2} ; r-1\right)$ & $n_{1}^{(+)}$ \\
& $D\left(E_{0}+\frac{1}{2}, j_{1}-\frac{1}{2}, j_{2} ; r-1\right)$ & $s_{1} n_{1}^{(-)}$ \\
& $D\left(E_{0}+\frac{1}{2}, j_{1}, j_{2}-\frac{1}{2} ; r+1\right)$ & $s_{2} n_{2}^{(-)}$ \\
& $D\left(E_{0}+\frac{1}{2}, j_{1}, j_{2}+\frac{1}{2} ; r+1\right)$ & $n_{2}^{(+)}$ \\
\hline 2 & $D\left(E_{0}+1, j_{1}, j_{2} ; r-2\right)$ & $n_{1}^{(+)} n_{2}^{(-)}$ \\
& $D\left(E_{0}+1, j_{1}+\frac{1}{2}, j_{2}+\frac{1}{2} ; r\right)$ & $n_{1}^{(+)} n_{2}^{(+)}$ \\
& $D\left(E_{0}+1, j_{1}+\frac{1}{2}, j_{2}-\frac{1}{2} ; r\right)$ & $s_{2} n_{1}^{(+)} n_{2}^{(-)}$ \\
& $D\left(E_{0}+1, j_{1}-\frac{1}{2}, j_{2}+\frac{1}{2} ; r\right)$ & $s_{1} n_{1}^{(-)} n_{2}^{(+)}$ \\
& $D\left(E_{0}+1, j_{1}-\frac{1}{2}, j_{2}-\frac{1}{2} ; r\right)$ & $s_{1} s_{2} n_{1}^{(-)} n_{2}^{(-)}$ \\
& $D\left(E_{0}+1, j_{1}, j_{2} ; r+2\right)$ & $n_{1}^{(-)} n_{2}^{(+)}$ \\
\hline 3 & $D\left(E_{0}+\frac{3}{2}, j_{1}, j_{2}+\frac{1}{2} ; r-1\right)$ & $n_{1}^{(+)} n_{1}^{(-)} n_{2}^{(+)}$ \\
& $D\left(E_{0}+\frac{3}{2}, j_{1}, j_{2}-\frac{1}{2} ; r-1\right)$ & $s_{1} n_{1}^{(-)} n_{2}^{(+)} n_{2}^{(-)}$ \\
& $D\left(E_{0}+\frac{3}{2}, j_{1}-\frac{1}{2}, j_{2} ; r+1\right)$ & $s_{2} n_{1}^{(+)} n_{1}^{(-)} n_{2}^{(-)}$ \\
& $D\left(E_{0}+\frac{3}{2}, j_{1}+\frac{1}{2}, j_{2} ; r+1\right)$ & $n_{1}^{(+)} n_{2}^{(+)} n_{2}^{(-)}$ \\
\hline 4 & $D\left(E_{0}+2, j_{1}, j_{2} ; r\right)$ & $n_{1}^{(+)} n_{1}^{(-)} n_{2}^{(+)} n_{2}^{(-)}$ \\
\hline & &
\end{tabular}


Table B.1: Multiplicities of $S U(2,2)$ representations in the decomposition of an $S U(2,2 \mid 1)$ unitary representation $\mathcal{D}\left(E_{0}, j_{1}, j_{2} ; r\right)$.

The non-chiral short multiplets are obtained by setting $j_{1}=j_{2}=j$, $E_{0}=2 j+1$ and $r=0$ so that $n_{1}^{(-)}=n_{2}^{(-)}=0$. The multiplet is shown in Table B.3. For a complete list of massless chiral and non-chiral short multiplets with spins not exceeding 2 , we refer the reader to, e.g., Table 5 in $[6]$.

\begin{tabular}{llll}
$E \backslash R$ & $r$ & $r-1$ & $r-2$ \\
\hline$E_{0}$ & $(j, 0)$ & & \\
$E_{0}+\frac{1}{2}$ & & $\left(j+\frac{1}{2}, 0\right) \oplus\left(j-\frac{1}{2}, 0\right)$ & \\
$E_{0}+1$ & & & $(j, 0)$
\end{tabular}

Table B.2: Chiral LH-multiplets $\mathcal{D}\left(E_{0}, j, 0 ; r\right)$, where $r=\frac{2}{3} E_{0}$.

\begin{tabular}{llll}
$E \backslash R$ & -1 & 0 & 1 \\
\hline$E_{0}$ & & $(j, j)$ & \\
$E_{0}+\frac{1}{2}$ & $\left(j+\frac{1}{2}, j\right)$ & $\left(j, j+\frac{1}{2}\right)$ \\
$E_{0}+1$ & & $\left(j+\frac{1}{2}, j+\frac{1}{2}\right)$ & \\
\hline
\end{tabular}

Table B.3: Non-chiral multiplets $\mathcal{D}\left(E_{0}, j, j ; 0\right)$, where $E_{0}=2 j+2$.

The multiplet with irrational $E_{0}$ found in section 6 is an example of a long representation with only nine $S U(2,2)$ representations because its vacuum representation has $j_{1}=j_{2}=0$.

Finally, there are multiplets with the simplest type of shortening when only one vanishing condition holds, so that the resulting multiplet extends through four energy levels, rather than three as in the case of the usual short multiplets. The defining vanishing relation for the modules of this type which arise in section 6 are: $E_{0}=2 j_{1}-\frac{3}{2} r+2$ or $E_{0}=2 j_{2}+\frac{3}{2} r+2$, which is the same as $n_{1}^{(-)}=0$ or $n_{2}^{(-)}=0$. We will refer to them as $\mathrm{RH}$ and LH semi-long multiplets. A typical LH-semi-long multiplet is of the form given in Table B.4. 


\begin{tabular}{lllll}
$E \backslash R$ & $r+1$ & $r$ & $r-1$ & $r-2$ \\
\hline$E_{0}$ & & $\left(j_{1}, j_{2}\right)$ & & \\
$E_{0}+\frac{1}{2}$ & $\left(j_{1}, j_{2}+\frac{1}{2}\right)$ & & $\left(j_{1}+\frac{1}{2}, j_{2}\right)$ & \\
& & & $\left(j_{1}-\frac{1}{2}, j_{2}\right)$ & \\
$E_{0}+1$ & & $\left(j_{1}+\frac{1}{2}, j_{2}+\frac{1}{2}\right)$ & & $\left(j_{1}, j_{2}\right)$ \\
$E_{0}+\frac{3}{2}$ & & $\left(j_{1}-\frac{1}{2}, j_{2}+\frac{1}{2}\right)$ & & \\
\hline
\end{tabular}

Table B.4: LH-semi-long multiplet $\mathcal{D}\left(E_{0}, j_{1}, j_{2} ; r\right)$, where $r=\frac{2}{3}\left(E_{0}-2 j_{2}-2\right)$.

The corresponding RH-semi-long multiplet is obtained from the above by $\left(j_{1}, j_{2}\right) \rightarrow\left(j_{2}, j_{1}\right)$ and $r \rightarrow-r$. Once more there might be a further shortening of semi-short multiplets for $j_{1}$ or $j_{2}$ equal zero. An example is the last multiplet in Table 6.2.

\section{APPENDIX C. $S O(7)$ and $S O(5,2)$ gamma matrices}

In this appendix we summarize explicit realizations of the $S O(7)$ and $S O(5,2)$ gamma matrices, which are needed to derive some of the formulae in section 6 and Appendix A.

\section{C.1. $S O(7)$ gamma matrices}

We follow here the same convention as in Appendix A of [6], where the $S O(7)$ gamma matrices, $\Gamma_{i}=\left(\Gamma_{i}^{a b}\right), i=0,1, \ldots, 6$, are hermitian and skew symmetric (i.e. pure imaginary) and satisfy

$$
\begin{gathered}
\left\{\Gamma_{i}, \Gamma_{j}\right\}=\delta_{i j}, \\
\Gamma_{0}=i \Gamma_{1} \Gamma_{2} \Gamma_{3} \Gamma_{4} \Gamma_{5} \Gamma_{5} .
\end{gathered}
$$

A particular realization with these properties is given by

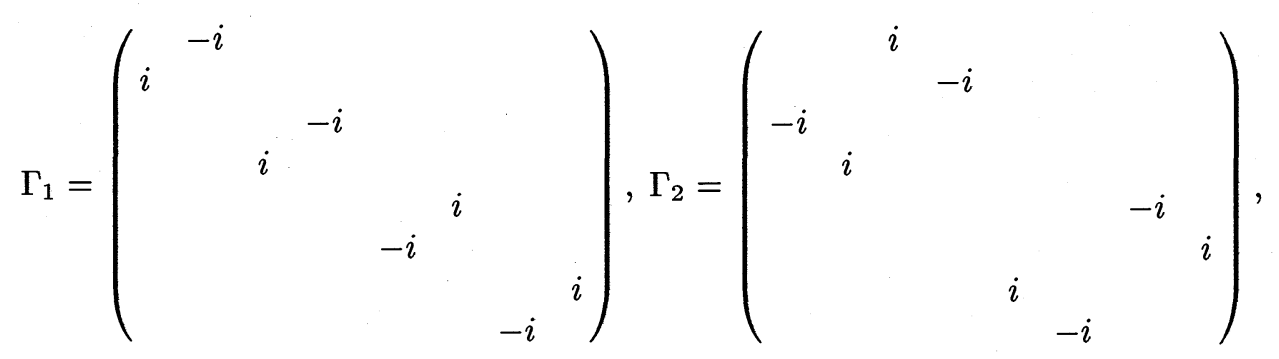




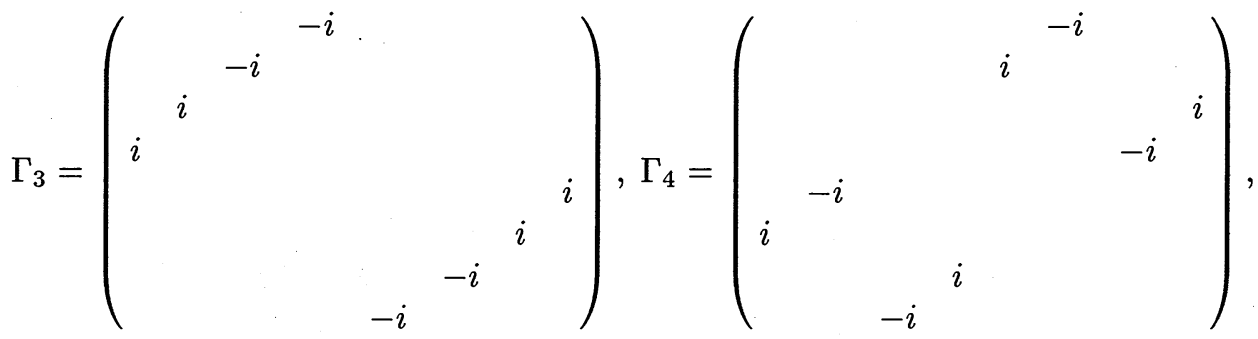

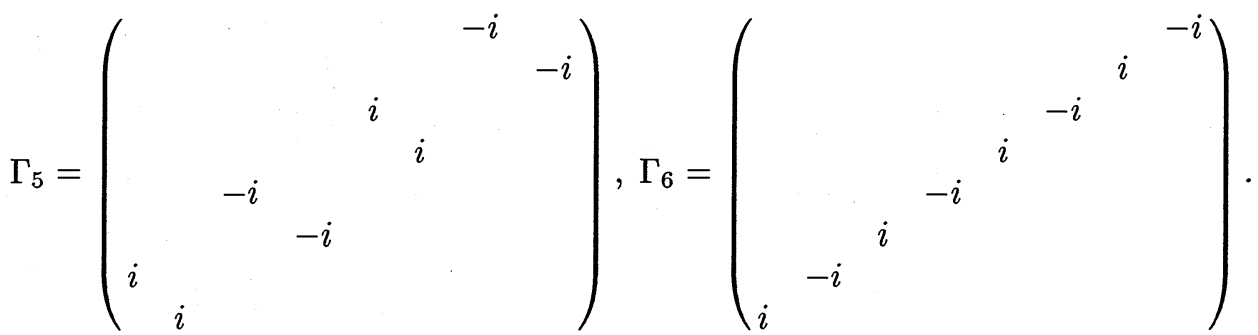

We also define

$$
\Gamma_{I J}=\frac{1}{2}\left(\Gamma_{I} \Gamma_{J}-\Gamma_{J} \Gamma_{I}\right)
$$

and

$$
\Gamma_{I \alpha}=\left(\Gamma_{I}, i \Gamma_{I} \Gamma_{0}\right)
$$

for $I, J=1, \ldots, 6$ and $\alpha=1,2$.

The $U S p(8)$ indices, $a, b, \ldots=1, \ldots, 8$, are raised and lowered using the symplectic form

$$
\Omega_{a b}=-\Omega^{a b}=i \Gamma_{0}^{a b}
$$

which is given by

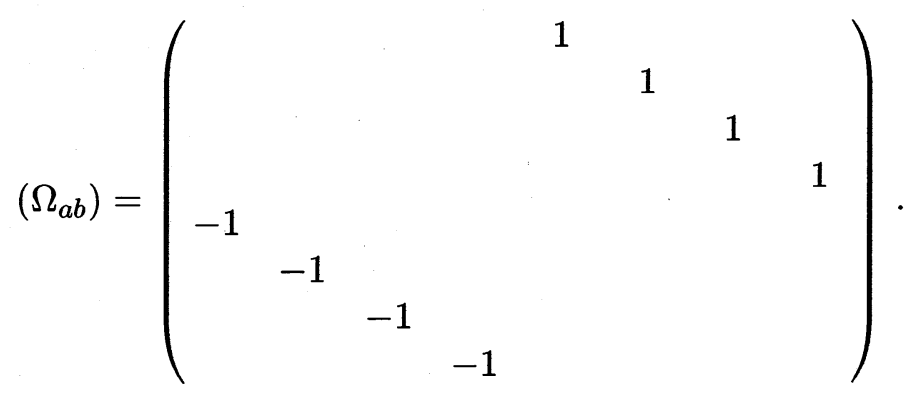

We refer the reader to [6] for further details.

C.2. $S O(5,2)$ gamma matrices

The $S O(5,2)$ gamma matrices, $\widetilde{\Gamma}_{i}, i=1, \ldots, 7$, satisfy

$$
\left\{\widetilde{\Gamma}_{i}, \widetilde{\Gamma}_{j}\right\}=2 \eta_{i j}
$$

where $\left(\eta_{i j}\right)=\operatorname{diag}(1,1,1,1,1,-1,-1)$. We use the following representation: 


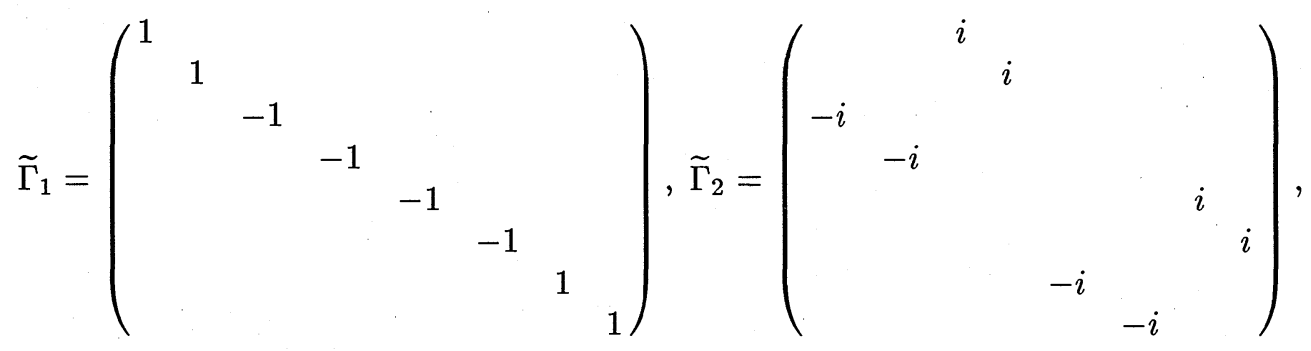

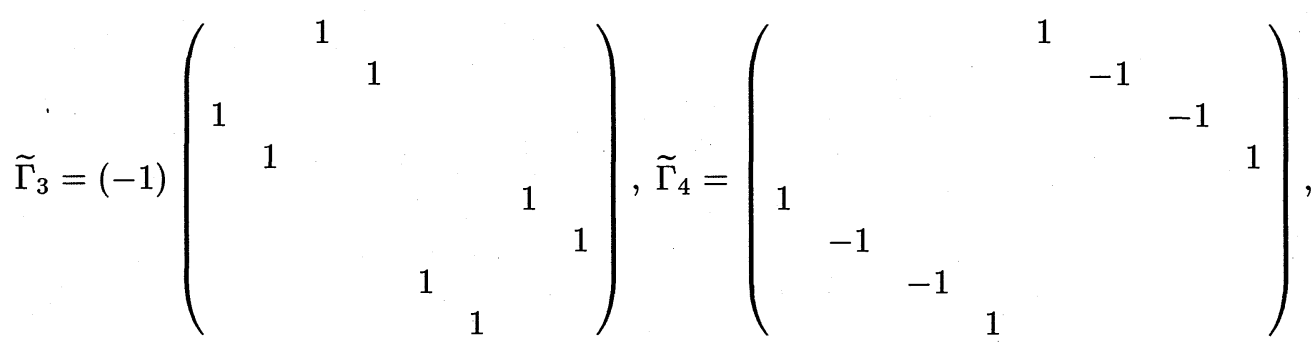

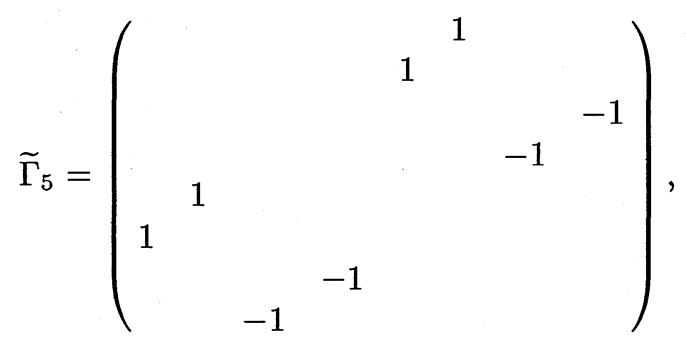

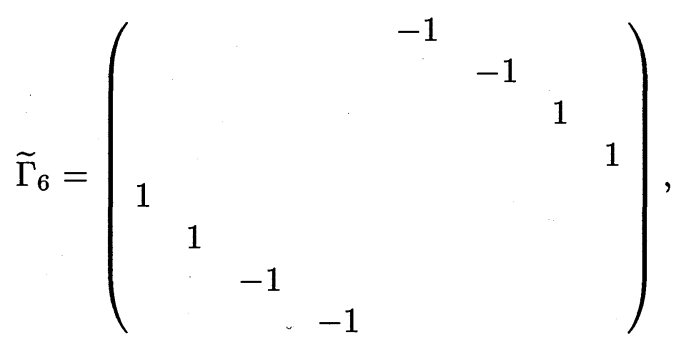

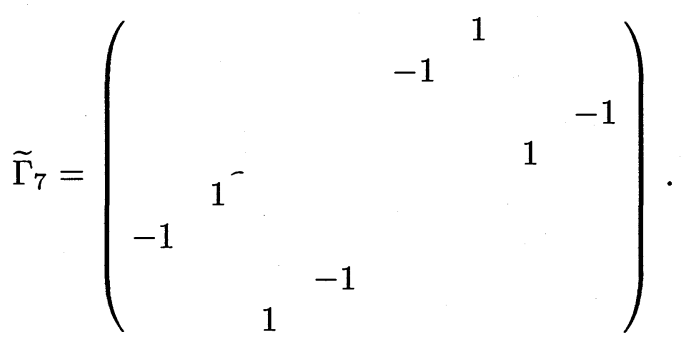

Since the spinor representation of $S O(5,2)$ is pseudoreal, we may define symplectic Majorana spinors, $\chi^{\alpha}, \alpha=1,2$. In the present realization they 
satisfy

$$
\chi^{\alpha}=-i \epsilon^{\alpha \beta} \widetilde{\Gamma}_{2}\left(\chi^{\beta}\right)^{*}
$$

where $\epsilon^{12}=1$.

\section{Acknowledgements}

We would like to thank A. Hanany, P. Mayr, J. Polchinski, S. Shenker, A. Strominger, and A. Zaffaroni for perspicacious commentary. The research of D.Z.F. was supported in part by the NSF under grant number PHY-9722072. The research of S.S.G. was supported by the Harvard Society of Fellows, and also in part by the NSF under grant number PHY-98-02709, and by DOE grant DE-FGO2-91ER40654. S.S.G. also thanks the Institute for Theoretical Physics at Santa Barbara, the University of Southern California, Stanford University, and the University of Pennsylvania for hospitality. The work of K.P. and N.W. was supported in part by funds provided by the DOE under grant number DE-FG03-84ER-40168. 


\section{References}

[1] J. Maldacena, The Large $N$ Limit of Superconformal Field Theories and Supergravity, Adv. Theor. Math. Phys. 2 (1998) 231 hep-th/9711200.

[2] S.S. Gubser, I.R. Klebanov, A.M. Polyakov, Gauge Theory Correlators from Non-Critical String Theory, Phys. Lett. B428 (1998) 105, hep-th/9802109.

[3] E. Witten, Anti-de Sitter space and holography, Adv. Theor. Math. Phys. 2 (1998) 253, hep-th/9802150.

[4] R. G. Leigh and M. J. Strassler, Exactly marginal operators and duality in four-dimensional $N=1$ supersymmetric gauge theory, Nucl. Phys. B447 (1995) 95-136, hep-th/9503121.

[5] M. Günaydin, L.J. Romans and N.P. Warner, Gauged $N=8$ Supergravity in Five Dimensions, Phys. Lett. 154B (1985) 268.

[6] M. Günaydin, L.J. Romans and N.P. Warner, Compact and NonCompact Gauged Supergravity Theories in Five Dimensions, Nucl. Phys. B272 (1986) 598.

[7] M. Pernici, K. Pilch and P. van Nieuwenhuizen, Gauged $N=8 \quad D=5$ Supergravity, Nucl. Phys. B259 (1985) 460.

[8] A. Khavaev, K. Pilch and N.P. Warner, New vacua of gauged $N=8$ supergravity in five-dimensions, hep-th/9812035.

[9] A. Karch, D. Lüst, and A. Miemiec, New N=1 superconformal field theories and their supergravity description, hep-th/9901041.

[10] N. Itzhaki, J. M. Maldacena, J. Sonnenschein, and S. Yankielowicz, Supergravity and the large $N$ limit of theories with sixteen supercharges, Phys. Rev. D58 (1998) 046004, hep-th/9802042.

[11] E. Witten, Anti-de Sitter space, thermal phase transition, and confinement in gauge theories, Adv. Theor. Math. Phys. 2 (1998) 505, hep-th/9803131.

[12] O. Aharony, M. Berkooz, D. Kutasov, and N. Seiberg, Linear dilatons, NS five-branes and holography, JHEP 10 (1998) 004, hep-th/9808149.

[13] I. R. Klebanov and A. A. Tseytlin, Asymptotic freedom and infrared behavior in the type 0 string approach to gauge theory, hep-th/9812089. 
[14] J. A. Minahan, Asymptotic freedom and confinement from type 0 string theory, hep-th/9902074.

[15] A. Kehagias and K. Sfetsos, On running couplings in gauge theories from type IIB supergravity, hep-th/9902125.

[16] S. S. Gubser, Dilaton driven confinement, hep-th/9902155.

[17] I. R. Klebanov and E. Witten, Superconformal field theory on threebranes at a Calabi-Yau singularity, Nucl. Phys. B536 (1998) 199, hep-th/9807080.

[18] S. S. Gubser, N. Nekrasov, and S. Shatashvili, Generalized conifolds and 4-dimensional $N=1$ superconformal field theory, hep-th/ 9811230.

[19] E. Lopez, A family of $N=1 S U(N)^{k}$ theories from branes at singularities, JHEP 2 (1998) 019, hep-th/9812025.

[20] L. Girardello, M. Petrini, M. Porrati and A. Zaffaroni, Novel Local CFT and Exact Results on Perturbations of $N=4$ Super YangMills from AdS Dynamics, JHEP 12 (1998) 022, hep-th/9810126. See hep-th version 4 or later, or the version published in JHEP, for discussion of the c-function.

[21] J. Distler and F. Zamora, Nonsupersymmetric Conformal Field Theories from Stable Anti-de Sitter Spaces, hep-th/9810206.

[22] S. S. Gubser, Einstein manifolds and conformal field theories, Phys. Rev. D59 (1999) 025006, hep-th/9807164.

[23] L.J. Romans, New Compactifications of Chiral N=2 D $=10$ Supergravity, Phys. Lett. 153B 392 (1985).

[24] P. van Nieuwenhuizen and N.P. Warner, New Compactifications of tendimensional and eleven-dimensional supergravity on manifolds which are not direct products, Commun. Math. Phys. 99141 (1985).

[25] M.R. Douglas and G. Moore, D-branes, Quivers, and ALE Instantons, hep-th/9603167.

[26] A. Lawrence, N. Nekrasov and C. Vafa, On conformal field theories in four-dimensions, Nucl. Phys. B533 (1998) 199, hep-th/9803015.

[27] V. Novikov, M. A. Shifman, A. I. Vainshtein, V. Zakharov, Exact Gell-Mann-Low Function of Supersymmetric Yang-Mills Theories from Instanton Calculus, Nucl. Phys. B229 (1983) 381. 
[28] M. Strassler, private communication.

[29] D. Anselmi, D. Z. Freedman, M. T. Grisaru, and A. A. Johansen, Nonperturbative formulas for central functions of supersymmetric gauge theories, Nucl. Phys. B526 (1998) 543, hep-th/9708042.

[30] I. I. Kogan, M. A. Shifman, and A. I. Vainshtein, Matching Conditions and Duality in N=1 SUSY Gauge Theories in the Conformal Window, Phys. Rev. D53 (1996) 4526, hep-th/9507170.

[31] M. Henningson, K. Skenderis, The Holographic Weyl Anomaly, JHEP 9807 (1998) 023, hep-th/9806087.

[32] S. S. Gubser and I. R. Klebanov, Absorption by branes and Schwinger terms in the world-volume theory, Phys. Lett. B413 (1997) 41.

[33] L. Susskind and E. Witten, The Holographic bound in anti-de Sitter space, hep-th/9805114.

[34] A. W. Peet and J. Polchinski, UV/IR relations in AdS dynamics, hep-th/9809022.

[35] L. Girardello, M. Petrini, M. Porrati, A. Zaffaroni, Confinement and condensates without fine tuning in supergravity duals of gauge theories, hep-th/9903026.

[36] R. M. Wald, General Relativity. Chicago University Press, Chicago (1984).

[37] A. B. Zamolodchikov, "Irreversibility" of the Flux of the Renormalization Group in a 2-d Field Theory, JETP Lett. 43 (1986) 730-732.

[38] D. Anselmi and A. Kehagias, Subleading corrections and central charges in the AdS/CFT correspondence, hep-th/9812092.

[39] O. Aharony, J. Pawełczyk, S. Theisen, and S. Yankielowicz, A note on anomalies in the AdS/CFT correspondence, hep-th/9901134.

[40] S. Forte and J. I. Latorre, A proof of the irreversibility of renormalization group flows in four dimensions, Nucl. Phys. B535 (1998) 709, hep-th/9805015.

[41] D. Anselmi, Anomalies, Unitarity and Quantum Irreversibility, hep-th/9903059.

[42] T. Appelquist, A. G. Cohen, and M. Schmaltz, A new constraint on strongly coupled gauge theories, hep-th/9901109. 
[43] E. Alvarez and C. Gomez, Geometric Holography, the Renormalization Group and the c-Theorem, Nucl. Phys. B541 (1999) 441, hep-th/9807226.

[44] E. Witten and D. Olive, Supersymmetry Algebras that include Topological Charges, Phys. Lett. 78B (1978) 97.

[45] M. Flato and C. Fronsdal, Representations of Conformal Supersymmetry, Lett. Math. Phys. 8 (1984) 159.

[46] V.K. Dobrev and V.B. Petkova, All Positive Energy Unitary Irreducible Representations of Extended Conformal Supersymmetry, Phys. Lett. 162B (1985) 127.

[47] E. Shuster, Killing spinors and Supersymmetry on AdS, hep-th/9902129.

[48] S. Ferrara, C. Fronsdal and A. Zaffaroni, On N=8 supergravity on AdS(5) and $N=4$ superconformal Yang-Mills theory, Nucl. Phys. $\mathbf{B 5 3 2}$ (1998) 153, hep-th/9802203.

[49] M. Henningson and K. Sfetsos, Spinors and the AdS/CFT correspondence, Phys. Lett. B431 (1998) 63, hep-th/9803251.

[50] W. S. l'Yi, Correlators of currents corresponding to the massive $p$ form fields in AdS/CFT correspondence, Phys. Lett. B448 (1999) 218, hep-th/9811097.

[51] G.E. Arutyunov and S.A. Frolov, Antisymmetric tensor field on $A d S^{5}$, Phys. Lett. B441 173 (1998), hep-th/9807046.

[52] P.K. Townsend, K. Pilch and P. van Nieuwenhuizen, Selfduality in odd dimensions, Phys. Lett. 136B (1984) 38.

[53] A. Volovich Rarita-Schwinger field in the AdS/CFT correspondence, JHEP 09 (1998) 022, hep-th/9809009.

[54] A.S. Koshelev and O.A. Rytchkov, Note on the massive RaritaSchwinger field in the AdS/CFT correspondence, hep-th/9812238.

[55] P. Breitenlohner and D.Z. Freedman, Positive Energy in anti-de Sitter Backgrounds and Gauged Extended Supergravity, Phys. Lett. 115B (1982) 197; Stability in Gauged Extended Supergravity, Ann. Phys. 144 (1982) 249.

[56] L. Mezincescu and P.K. Townsend, Stability at a Local Maximum in Higher Dimensional anti-de Sitter Space and Applications to Supergravity, Ann. Phys. 160 (1985) 406. 
[57] D. Z. Freedman, S. D. Mathur, A. Matusis, and L. Rastelli, Correlation functions in the $C F T_{D} / A d S_{D+1}$ correspondence, hep-th/9804058.

[58] S. Ferrara and A. Zaffaroni, $N=1, N=2$ 4-D superconformal field theories and supergravity in $A d S_{5}$, Phys. Lett. B431 (1998) 49, hep-th/9803060.

[59] S. Ferrara, M.A. Lledo and A. Zaffaroni, Born-Infeld corrections to D3-brane action in $A d S_{5} \times S_{5}$ and $N=4, d=4$ primary superfields, Phys. Rev. D58 (1998) 105029, hep-th/9805082.

[60] E. Cremmer, Supergravities in Five Dimensions, in Superspace and Supergravity, S.W. Hawking and M. Roček (editors), Proceedings of the Nuffield Gravity Workshop, Cambridge, Jun 16 - Jul 12, 1980; Cambridge Univ. Pr. (1981) .

[61] M. Awada and P.K. Townsend, $N=4$ Maxwell Einstein Supergravity in Five Dimensions and its SU(2) Gauging, Nucl. Phys. B255 (1985) 617 .

[62] L.J. Romans, Gauged N=4 Supergravities in Five-Dimensions and Their Magnetovac Backgrounds, Nucl. Phys. B267 433 (1986).

[63] S. Kachru and E. Silverstein, 4-D Conformal Theories and Strings on Orbifolds, Phys. Rev. Lett. 80 (1998) 4855, hep-th/9802183.

[64] S. Helgason, Differential Geometry and Symmetric Spaces, Academic Press, New York (1962).

[65] V. Kac and M. Wakimoto, Integrable highest weight modules over affine superalgebras and number theory, in Lie Theory and Geometry, J. Brylinski et al. (eds.), Birkhauser, Boston (1994).

[66] S. Ferrara, R. Kallosh, and A. Strominger, $N=2$ extremal black holes, Phys. Rev D52 (1995) 5412.

[67] M. Cvetic and H. H. Soleng, Supergravity domain walls, Phys. Rept. 282 (1997) 159. 